\title{
Derivation of Classical Mechanics in an Energetic Framework via Conservation and Relativity
}

\author{
Philip Goyal* \\ University at Albany (SUNY), NY, USA
}

(Dated: April 18, 2020)

\begin{abstract}
The concepts of conservation and relativity lie at the heart of classical mechanics. In the hands of Descartes, Galileo, and Huygens, these concepts led to early results which were critical to its early development. However, over the following two centuries, their perceived importance to the structure of mechanics underwent considerable change. In view of this complex historical development, to what extent do conservation and relativity determine the structure of mechanics? In this paper, we address this question by deriving classical mechanics - both nonrelativistic and relativistic - using relativity and conservation as the primary guiding principles. The derivation proceeds in three distinct steps. First, conservation and relativity are used to derive the asymptotically conserved quantities of motion. Second, in order that energy and momentum be continuously conserved, the mechanical system is embedded in a larger energetic framework containing a massless component that is capable of bearing energy (as well as momentum in the relativistic case). Imposition of conservation and relativity then results, in the nonrelativistic case, in the conservation of mass and in the frame-invariance of massless energy; and, in the relativistic case, in the rules for transforming massless energy and momentum between frames. Third, a force framework for handling continuously interacting particles is established, wherein Newton's second law is derived on the basis of relativity and a staccato model of motion-change. Finally, in light of the derivation, we elucidate the structure of mechanics by classifying the principles and assumptions that have been employed according to their explanatory role, distinguishing between symmetry principles and other types of principles (such as compositional principles) that are needed to build up the theoretical edifice.
\end{abstract}

\section{INTRODUCTION}

Two key concepts, namely conservation and relativity, lie at the heart of classical mechanics. Each rests on a compelling idea - conservation on the idea that change is underlain by changelessness; relativity on the idea that, although observations are necessarily perspectival, there are classes of observers which are, in some fundamental sense, physically equivalent.

The power of these concepts, both separately and conjointly, was demonstrated in the early development of mechanics. Descartes formalized the notion of conservation though the principle that a system of colliding bodies conserves its total scalar 'quantity of motion', a principle that guided the formulation of his laws of collision $^{2}$. Similarly, Galileo's principle of relativity enabled

\footnotetext{
*Electronic address: pgoyal@albany.edu

${ }^{1}$ In Principles II 36 [1], Descartes asserts: "there is a fixed and determined quantity of [motion] ... always the same in the universe as a whole even though there may at times be more or less motion in certain of its individual parts", and that "when one part of matter moves twice as fast as another twice as large, there is as much motion in the smaller as in the larger", roughly interpreted as the assertion that $\sum_{i} m_{i} u_{i}$ is the conserved quantity, where $m$ is a measure of the 'size' of a body.

2 Descartes' conservation principle was insufficient to account for collisional behaviour. Lacking another principle of similar scope
}

his derivation of parabolic motion from vertical free fall, and later enabled Huygens' deduction of the behaviour of equal bodies in head-on elastic collision. Huygens went even further, showing that one could combine conservation (in the form of a generic principle of the conservation of a scalar quantity of motion) and relativity to derive a new conservation law, namely the conservation of relative speed, applicable to unequal bodies in head-on collision ${ }^{3}$.

capable of rectifying this insufficiency, Descartes introduced other considerations in a rather ad hoc manner. The defects of the resultant laws of collision were readily apparent. For example, Leibniz showed these laws to be inconsistent with the requirement of continuity [2, pp. 290-291]. Nevertheless, these laws were a spur to development of the correct laws.

3 Huygens' laws of collisions can be be divided into two cases: (i) For equal bodies in head-on collision (whether elastic or not), all collisions involving bodies with unequal incident speeds follows via relativity from the case of equal incident speeds, the behaviour in this latter case being taken as axiomatic. (ii) For unequal bodies in head-on elastic collision, the additional assumption of the asymptotic conservation of total scalar quantity of motion, where the quantity of motion is a function of speed, and an auxiliary assumption (if one mass undergoes a change, so must the other) implies that the relative speed of the two masses is the same before and after the collision. Proof sketch: in any such a collision, there is a Galilean frame of reference in which the speed of one body does not change (comparing the initial and final states), only its direction of motion. Hence, its quantity of motion does not change. But, asymptotic conservation of total quantity of motion then implies that the speed of the other body 
Despite their fecundity, the notions of conservation and relativity were eclipsed in Newton's theory of mechanics by the notion of force, with a specific law-Newton's second law-placed at its centre. The reasons for this are two-fold.

First, in the process of being formalized and applied to the task of formulating laws of collision, Descartes' conservation principle was confronted with a number of challenges which brought into question not only its mathematical form, but also its range of applicability and the validity of its conceptual justification:

1. Mathematical form of the quantity of motion. Descartes' choice of the conserved quantity of motion, namely, $m v$, was dictated by mathematical simplicity, not by a physical principle. This fact was brought into focus by Huygens, who showed that Descartes' $m v$ was incompatible with relativity, and further that, granted other established physical laws and principles, $m v^{2}$ (known after Leibniz [5] as vis viva) was the correct scalar quantity of motion ${ }^{4}$.

2. Elastic collisions as continuous processes. Descartes viewed matter as pure extension, and collisions accordingly as instantaneous events between rigid geometric figures. In contrast, Newton and Leibniz insisted of the continuity of most natural processes, and accordingly viewed an elastic collision as a finite process involving deformation. But, in such a process, the bodies would be momentarily stilled in some reference frame. Thus, any principle positing the conservation of a total scalar quantity of motion could only apply to the collision's asymptotic states.

3. Dissipation of motion in atomic collisions. Newton (amongst others) believed that atoms in head-on collision would lose their motion ${ }^{5}$, an idea that con-

also does not change. But if the direction of one mass changes, so must the other (by the auxiliary assumption). Hence, relative speed in this chosen frame is same before and after. But relative speed is frame-independent. Therefore, irrespective of the (inertial) frame in which the collision is viewed, the relative speed is unchanged. For details, see [3, pp. 313-317] and also [4, §9.4].

4 Huygens' law of conservation of relative speed of two bodies in head-on elastic collision (see footnote 3 ) implies that the conserved quantity of motion cannot be $m v$ (as can be seen by considering a body of mass $m<M$ striking a body of mass $M$ initially at rest). Furthermore, appealing to Galileo's law of free fall and Torricelli's principle (that the centre of gravity of a system of interacting bodies cannot rise), Huygens showed that the conserved quantity of motion is, in fact, $m v^{2}$.

5 Newton (amongst others) asserted that atoms were hard bodies that collide completely inelastically [6, pp. 4-5]. Hence the fundamental importance of formulating laws applicable to inelastic collisions. flicted with the intuition underpinning Descartes' conservation principle (see footnote 1).

The hypothesized dissipation of motion during the collision of hard atoms, and the requirement of continuity for elastic collisions, resulted in a marginalization of scalar conservation principles ${ }^{6}$, and lead - via a vectorialization of Descartes' conservation principle (due to Wren [9], Huygens [10, 11], Wallis [12], and Newton) - to a new conservation principle, namely the principle of conservation of momentum. However, this vectorialization severed the connection between the mathematical principle and Descartes' intuitive motivation for his principle; and a novel justification for the new principle was not readily forthcoming ${ }^{7}$.

Second, the challenge of expanding mechanics beyond collisional phenomena to encompass bodies continuously interacting at a distance made clear that new ideas or principles, beyond relativity and conservation, were required. Newton's framework, organized around the concept of force, provided the key new idea, namely a specific law-Newton's second law-relating a body's acceleration with the force acting upon it. Conservation of momentum was recast as a constraint on the forces exerted by two bodies upon one another (the principle of action and reaction), which also thereby provided some kind of intuitive justification for momentum conservation; whilst relativity was recast as the requirement that force be independent of inertial frame. Thus, conservation and relativity were subsumed within the framework, with a specific law placed at its centre.

However, a number of developments in the nineteenth and early twentieth century brought the general principles of conservation and relativity once again firmly into the foreground:

1. Interconversion phenomena. Following the discovery of new interconversion phenomena in the first third of the nineteenth century, a scalar conservation principle, the conservation of energy, arose to fill the need for a quantitive means to coordinate these diverse (electrical, magnetic, thermal, mechanical, and chemical)

${ }^{6}$ Although Leibniz championed the conservation of vis viva, a compelling account of the 'missing' quantity of motion at the stillpoint of an elastic collision, or at the end-point of an inelastic collision, was lacking. As a consequence, scalar conservation principles were marginalized. For example, in textbooks through to the end of the eighteenth century, elastic collisions were handled by using a situation-specific law (Huygens' conservation of the masses' relative speed - see Footnote 3), rather than the asymptotic conservation of vis viva — see [7] (Appendix) and [8].

7 Some attempts were made to justify the mathematical principle of momentum conservation in terms of the law of the lever. See, for example, [9], and [2, pp. 203-206]. 
phenomena [13]. During this period, mechanics was regarded as a component of a larger energetic framework, which allowed for the interconversion of energy of motion-quantified by vis viva - and non-motive forms of energy.

2. Principled derivation of mechanics. During the nineteenth century, there were numerous attempts to derive key features of Newtonian mechanics using general physical principles, such as relativity. For example, Laplace and Bélanger offered novel derivations of Newton's second law ${ }^{8}$, while Schütz used relativity to derive momentum conservation from energy conservation [15].

3. Interpretation of Maxwell's equations. In the last third of the nineteenth century, the interpretation of Maxwell's equations in terms of a privileged frame of reference brought the validity of the principle of relativity (and hence Newtonian mechanics) into question. Einstein's special relativity not only rescued Galileo's principle of relativity from this doubt, but, through the derivation of a new kinematics and dynamics, demonstrated anew its fecundity.

By the close of the foregoing developments, the energetic framework (extended to include massless momentum), with its conservation laws, had become established as an indispensable means to coordinate the distinct physical theories of mechanical, electromagnetic, and thermal phenomena which had been formulated.

As this sketch illustrates, the relationship between mechanics and the notions of relativity and conservation has followed a long and complex evolutionary path. This raises the question: what is the degree to which these notions determine the structure of mechanics, particularly if the notion of an energetic framework is granted? In particular, is it possible, in retrospect, to build up mechanics - both nonrelativistic and relativistic - in a systematic manner based on the notions of conservation and relativity? And, if so, what additional ideas and principles are required, and what insights are thereby obtained?

In this paper, we address these question by building up classical mechanics - both nonrelativistic and relativistic - using the principle of relativity and the notion of conservation as the primary guiding ideas. This is carried out in three distinct steps ${ }^{9}$.

\footnotetext{
${ }^{8}$ See Ref. [14] for a detailed historical investigation into these derivations.

9 These steps - and the principles employed and results obtainedare summarized in Tables I and II (pages 20 and 21).
}

1. Asymptotic conservation. First, by considering a specific symmetric collision, we derive the mathematical forms of the asymptotically conserved quantities of motion by appeal to the principle of relativity. Here, functional equations are the key to harnessing of the spirit of Huygens' arguments to obtain the mathematical form of the quantities of motion. An argument due to Schütz is used to derive asymptotic momentum conservation from asymptotic energy conservation.

2. Energetic framework. Next, we embed a system of interacting bodies in an energetic framework containing a massless component that can bear energy (and, in the relativistic case, momentum). This framework allows for the passage of energy (and possibly momentum) between its massive component (consisting of bodies in motion) and its massless component. Relativity and conservation are then used to determine (i) which interconversions are possible, and (ii) how the energy (and, in the relativistic case, the momentum) associated with the massless component transforms between inertial frames.

3. Staccato model of motion change. Finally, we posit a specific model of how a body undergoes change of motion due to the influence of another. Using this model, Newton's second law is derived via relativity.

By building up mechanics in this layered manner, the distinct types of principles out of which mechanics is built up - ranging from the most general to the most specific become clearly visible. For example, in addition to conservation and relativity, we find that compositional principles play a fundamental role. It also becomes apparent that meta-theoretic desiderata, such as continuity, play a pivotal role. For example, in our development, the move from the first step to the second, where (in the nonrelativistic case) a massless form of energy is posited, is driven by the desideratum that total energy be continuously - not just asymptotically - conserved. Similarly, in the relativistic case, a massless form of momentum must be posited in order that momentum be continuously conserved.

By approaching mechanics using the notion of the energetic system, certain results are obtained with surprising ease. For example, whereas the energy-momentum transformations laws for an electromagnetic system are ordinarily derived by consideration of the stress-energymomentum tensor [16] (see also [17, 18]), we find that, in the context of the energetic framework, conservation and relativity jointly imply that massless energy and momentum transform as a four-vector, without recourse to any specific model of the massless component. In addition, in the nonrelativistic case, the corresponding argument 
shows that massless energy is frame-invariant, which has the perhaps surprising consequence that the nonrelativistic kinetic theory of gases is inconsistent, consistency only achievable in the relativistic case $\mathrm{e}^{10}$.

Finally, since our approach depends primarily on relativity and conservation, the parallelism between nonrelativistic and relativistic mechanics can be clearly exhibited. The shift from nonrelativistic to relativistic mechanics is straightforwardly achieved by changing the kinematical group by which relativity is implemented, and by allowing the massless component of the energetic system to bear momentum as well as energy. The possibility of interconversion between rest energy and other energetic forms directly follows from these changes, without the customary appeal to other special considerations (such as the laws governing the behaviour of photons).

Previous efforts to derive classical mechanics using relativity (and, to varying degrees, conservation) have tended to focus on deriving parts of mechanics, taking the remainder as given-for example, on deriving the conserved quantities of motion (reviewed in Sec. IV), or on deriving Newton's second law [14] - rather than on deriving the whole, as is done here. In particular, our introduction of an energetic framework (in Step 2, above) appears to be novel, providing a vital conceptual interface between the asymptotically conserved quantities and the force framework.

The paper is organized as follows. In Sec. II, we derive nonrelativistic mechanics in an energetic framework, beginning with the derivation of the nonrelativistic quantities of motion (Sec. II A), and then building up the energetic framework (Sec. IIB). The development of the Newtonian framework as a subset of the resulting structure, and the insights to which this leads, are described in Sec. II C. A parallel treatment of relativistic mechanics is carried out in Sec. III. In Sec. IV, we briefly describe and analyze a selection of other derivations of quantities of motion from the literature.

In Sec. V, we clarify the structure of classical mechanics in light of our derivation by classifying and analyzing the physical principles employed, and examining the insights that our approach provides about the subtle issues mentioned above that arose in the historical development of mechanics. We conclude in Sec. VI with a discussion

10 Insofar as heat is regarded as a form of massless energy, it is a frame-invariant quantity (in the nonrelativistic case). Hence, it cannot be represented by the kinetic energy of a set of bodies in motion, which is not frame-invariant. This difficulty vanishes in the relativistic case, wherein both massless and massive energymomentum transform in the same manner. of the relation between symmetry principles and conservation laws, and of pedagogical approaches to mechanics, in light of the present derivation.

\section{NONRELATIVISTIC MECHANICS}

\section{A. Conserved quantities of motion}

\section{Derivation of particle energy as asymptotically conserved scalar quantity of motion}

According to Descartes' conservation principle, the total (scalar) quantity of motion is conserved in any elastic collision. However, if one supposes that a body's motion changes continuously, it follows that the bodies in elastic collision can be momentarily stilled. Thus, one can only hope to conserve the total quantity of motion if one compares a collision's pre- and post-collisional states. We now derive the form of that quantity by considering a specific collision which is observed from two different inertial frames, namely the lab frame, $S$, and a moving frame, $S^{\prime}$.

We assume that a particle of $\operatorname{mass}^{11} m$ with speed $u$ has a scalar quantity of motion $f_{m}(u)$, to which we henceforth refer as its energy. By hypothesis, this function $f$ is independent of the specific situation in which the particle finds itself. Hence, if we can determine the form of $f$ by considering specific situations that we presume to be possible, then that form of $f$ must apply to all situations. We further assume that the total energy of a system of widely-separated particles is the sum of their separate energies $^{12}$.

Suppose that, as observed from inertial frame $S$, two particles of equal mass approach from opposite directions, moving at the same speed, $u$, along the $x$-axis, and collide elastically at the origin, $\mathrm{O}$ (see Fig. 1) ${ }^{13}$. We assume that it is possible for the particles, after collision, to recede in opposite directions along the y-axis with their speeds undiminished ${ }^{14}$. Suppose that frame $S^{\prime}$ moves

11 The mass of a body is here assumed to be independent of the body's state of motion, and hence frame-independent.

12 This energy-additivity is in fact derivable from the weaker assumption of associativity (see Sec. VB).

13 We assume here that isolated bodies move at constant velocity.

14 The possibility of post-collisional motion along the $y$-axis can be traced to the requirement of continuity together with the fact that both grazing and head-on collisions are possible. The possibility that, additionally, the post-collisional speeds coincide with the pre-collisional speeds then follows from the assumptions that (i) relatively-rotated reference frames are physically equivalent, and (ii) the time-reversed version of an elastic collision (viz. a collision that asymptotically conserves the total scalar quantity 


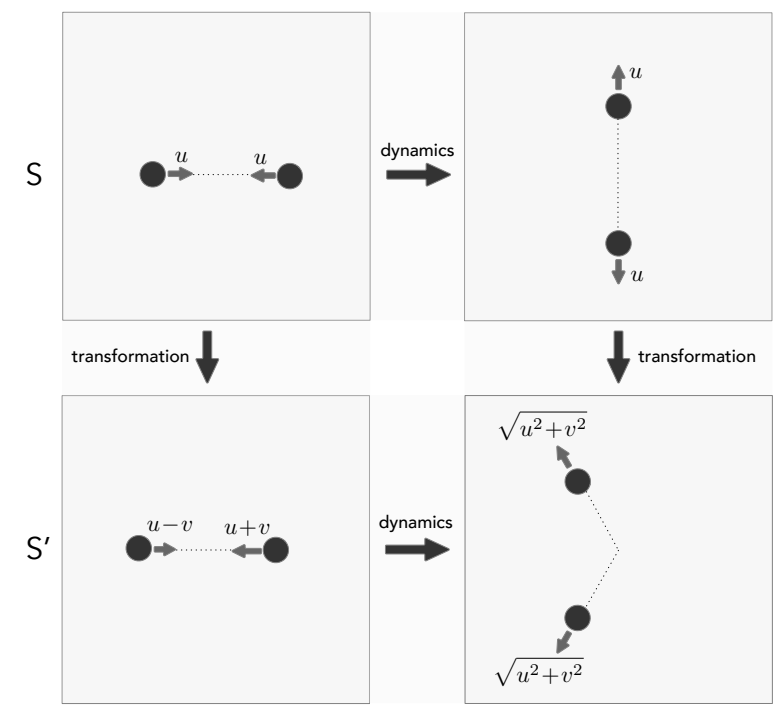

FIG. 1: Derivation of nonrelativistic particle energy via asymptotic scalar conservation and relativity. Consider an elastic collision of two equal-mass particles, as viewed in frames $S$ and $S^{\prime}$. In frame $S$, the particles, each of mass $m$, approach at equal speed $u$, and recede along the $y$-axis at the same speeds. In this frame, the total pre- and post-collisional energy of the system (assumed, for widely-separated particles, to be sum of the energies of the separate particles) is trivially conserved since the pre- and post-collisional speeds are the same. By relativity, frames $S$ and $S^{\prime}$ are physically equivalent, so that, if the situation in $S$ is physically possible (as is here assumed), the situation in $S^{\prime}$ is also. Therefore, since energy is asymptotically conserved in $S$, it must also be conserved in $S^{\prime}$. That condition leads to a functional equation, $f_{m}(u+v)+f_{m}(u-v)=2 f_{m}\left(\sqrt{u^{2}+v^{2}}\right)$, for the energy, $f_{m}(u)$, of a particle of mass $m$. Its solution is $f_{m}(u)=a u^{2}+b$, where $a, b$ are functions of $m$.

at speed $v$ along the $x$-axis of $S$. Since asymptotic energy conservation trivially holds in frame $S$, and since frames $S$ and $S^{\prime}$ are (by relativity) physically equivalent, asymptotic energy conservation must also hold in frame $S^{\prime}$, which leads to the condition, for all $u \geq 0$ and all $v \in[-u, u]$,

$$
f_{m}(u+v)+f_{m}(u-v)=2 f_{m}\left(\sqrt{u^{2}+v^{2}}\right),
$$

whose general solution is

$$
f_{m}(u)=a(m) u^{2}+b(m)
$$

where $a, b$ are undetermined functions of $m$ (see Appendix A 1).

To determine the forms of functions $a(m)$ and $b(m)$,

of motion) is also possible. assume that an object of mass $m$ moving at speed $u$ can equally be regarded as a composite ${ }^{15}$ of two noninteracting masses, $m_{1}$ and $m_{2}$, such that $m=m_{1}+m_{2}$, moving together at speed $u$. This composite has total energy

$$
f_{m_{1}}(u)+f_{m_{2}}(u)=\left[a\left(m_{1}\right)+a\left(m_{2}\right)\right] u^{2}+\left[b\left(m_{1}\right)+b\left(m_{2}\right)\right] .
$$

Thus, for any $m_{1}, m_{2}$ and any $u$,

$$
\begin{aligned}
a\left(m_{1}+m_{2}\right) u^{2}+b\left(m_{1}+m_{2}\right)= & {\left[a\left(m_{1}\right)+a\left(m_{2}\right)\right] u^{2} } \\
& +\left[b\left(m_{1}\right)+b\left(m_{2}\right)\right] .
\end{aligned}
$$

Therefore, functions $a(m)$ and $b(m)$ both obey Cauchy's additive functional equation,

$$
\begin{aligned}
& a\left(m_{1}+m_{2}\right)=a\left(m_{1}\right)+a\left(m_{2}\right) \\
& b\left(m_{1}+m_{2}\right)=b\left(m_{1}\right)+b\left(m_{2}\right),
\end{aligned}
$$

which have general solutions $a(m)=\alpha m$ and $b(m)=$ $\beta m$, where $\alpha, \beta$ are numerical constants. Hence,

$$
f_{m}(u)=\alpha m u^{2}+\beta m .
$$

The kinetic energy is $\alpha m u^{2}$, and the rest energy $\beta m$. As the energy-scale is arbitrary up to a multiplicative factor, we can set $\alpha=1 / 2$ to conform with convention.

\section{Derivation of particle momentum as asymptotically conserved vectorial quantity of motion}

According to Newton's action-reaction principle, in a two-body system, any change in the motion of one body is accompanied by a corresponding change in the motion of the other, in such a way that the total change in motion (when suitably quantified) is zero if the direction of the change is taken into account. Accordingly, we assume that there exists a vector quantity of motion whose (vector) sum for a system of bodies in elastic collision is conserved if one considers the pre- and post-collisional states. We now derive the form of this conserved quantity in two ways: (1) by considering an elastic collision as observed from two different frames; (2) by using an argument due to Schütz [15].

1. Momentum via elastic collision. Consider the elastic collision of Sec. II A 1. Let the vectorial quantity of motion of a particle moving with velocity $\mathbf{u}$ be denoted $g(u) \hat{\mathbf{u}}$, and assume that the total vectorial quan-

15 That the mass of the composite is equal to the sum $m_{1}+m_{2}$ can either be assumed, or derived from the weaker assumption of associativity (see Sec. VB). 
tity of motion is the vectorial sum of the individual particles' vectorial quantity of motion. Applying conservation of total vectorial quantity of motion in frame $S^{\prime}$ as resolved along the $x^{\prime}$-axis, we obtain, for all $u \geq 0$ and all $v \in[-u, u]$,

$$
g(v+u)-g(v-u)=2 g(w) \cdot \frac{v}{w},
$$

where $w=\sqrt{u^{2}+v^{2}}$. As shown in Appendix A 2, this equation has general solution

$$
g(u)=a^{\prime}(m) u
$$

where $a^{\prime}(m)$ is an undetermined function.

Thus, the vectorial quantity of motion associated with a particle of mass $m$ moving with velocity $\mathbf{u}$ is $g(u) \hat{\mathbf{u}}=$ $a^{\prime}(m) \mathbf{u}$. Consideration of a mass $m$ as a composite of masses $m_{1}$ and $m_{2}$ as in Sec. II A 1, above, implies that $a^{\prime}(m)=\alpha^{\prime} m$, where $\alpha^{\prime}$ is a numerical constant. Hence, a mass $m$ moving at speed $u$ has vectorial quantity of motion

$$
g_{m}(u) \hat{\mathbf{u}}=\alpha^{\prime} m \mathbf{u} .
$$

Following convention, we set $\alpha^{\prime}=1$, yielding the momentum $m \mathbf{u}$.

Remark. One might imagine considering the initial state and stillpoint of an elastic head-on collision between two equal masses initially travelling at equal speed $u$. Conservation of the vectorial quantity of motion in frame $S^{\prime}$ would presumably then yield the equation $g(v+u)-$ $g(v-u)=2 g(v)$, with solution $g(u)=a^{\prime}(m) u$. However, since conservation of energy in $S^{\prime}$ implies that there is some non-motive energy present at the stillpoint, one is here making an implicit assumption, namely that there is no momentum associated with this non-motive energy. While this happens to be true in the nonrelativistic case, it is not true in the relativistic one. More importantly, this (implicit) assumption constitutes an assumption about the larger energetic framework (see Sec. II B), which deserves considered justification. Hence, in deriving the form of the vectorial quantity of motion, it is advisable to consider only the asymptotic states of an elastic collision, where (since the initial and final motive energies are the same) no such assumption is required.

2. Momentum via Schütz's argument It is also possible to derive the form of the vectorial conserved quantity as a direct consequence of asymptotic energy conservation and relativity. Consider a general elastic collision in which, in frame $S^{\prime}$, two objects of mass $m_{1}$ and $m_{2}$ undergo head on collision with initial velocities $\mathbf{u}_{1}, \mathbf{u}_{2}$, and separate at velocities $\mathbf{u}_{1}^{\prime}, \mathbf{u}_{2}^{\prime}$. Energy conservation in frames $S$ and $S^{\prime}$ implies that

$$
m_{1} u_{1}^{2}+m_{2} u_{2}^{2}=m_{1} u_{1}^{\prime 2}+m_{2} u_{2}^{\prime 2}
$$

and that, for all $\mathbf{v}$,

$$
\begin{aligned}
m_{1}\left|\mathbf{u}_{1}+\mathbf{v}\right|^{2}+m_{2}\left|\mathbf{u}_{2}+\mathbf{v}\right|^{2}=m_{1} \mid \mathbf{u}_{1}^{\prime} & +\left.\mathbf{v}\right|^{2} \\
& +m_{2}\left|\mathbf{u}_{2}^{\prime}+\mathbf{v}\right|^{2} .
\end{aligned}
$$

Subtracting the foregoing equations,

$$
m_{1} \mathbf{u}_{1}+m_{2} \mathbf{u}_{2}=m_{1} \mathbf{u}_{1}^{\prime}+m_{2} \mathbf{u}_{2}^{\prime}
$$

which is momentum conservation. It follows that the vectorial quantity of motion associated with a particle of mass $m$ moving at velocity $u$ is $\alpha m \mathbf{u}$ up to an additive vectorial constant. Consideration of the elastic collision above shows that the vectorial constant is zero.

\section{B. Continuous conservation of energy and momentum in the energetic framework}

We have earlier noted that conservation of a total scalar quantity of motion can only hold asymptotically, and only then for elastic collisions. In order to generalize this conservation law so that it applies continuously, and to all collisions, we must posit that every system of bodies exists within a larger energetic framework that can contain a massless component capable of bearing energy.

Since the imposition of continuous momentum conservation encounters no obvious obstacles when applied to bodies undergoing inelastic collisions, there is no specific need to assume that the massless component is also capable of bearing momentum.

The question then arises as to what kinds of interconversions of rest energy, kinetic energy, and massless energy are possible, and how the energy of the massless component transforms between frames. By requiring continuous conservation of total energy and momentum, and by imposing relativity, we shall see that (see Fig. 2):

1. System mass is conserved. Therefore, rest energy cannot be dynamically converted into kinetic energy or massless energy.

2. Interconversion of kinetic energy and massless energy is permitted.

3. Massless energy takes the same value in all frames.

4. If two states - possibly of different systems - have equal values of total mass, energy, and momentum as observed in frame $S$, then this equality holds 


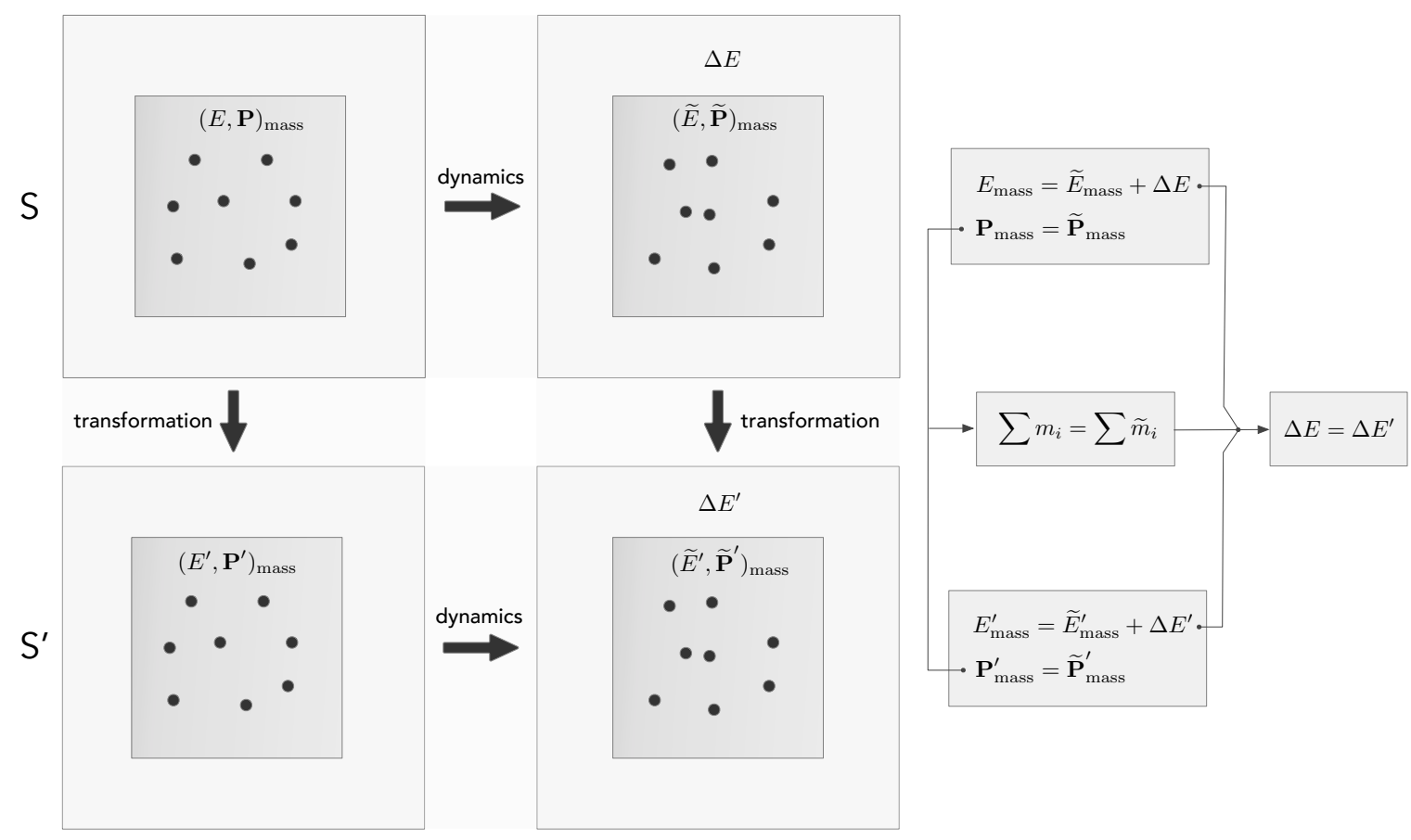

FIG. 2: Mass conservation and frame-invariance of massless energy in the energetic framework. The energetic framework posits that, in addition to a system of bodies with their energies and momenta, there exists a massless component capable of bearing energy. Within such a framework, energy can be continuously (not just asymptotically) conserved. In this example, in frame $S$, we consider a process in which the system initially has no massless energy, but dynamically evolves to a state in which the massless energy is $\Delta E$. If conservation of total energy and momentum is applied to this dynamical process as seen in frames $S$ and $S^{\prime}$ (Eqs. (8), (9), (11), and (12)), one finds that momentum conservation implies total mass conservation (Eq. (10)), and energy conservation then implies (Eq. (13)) the frame-invariance of massless energy, $\Delta E=\Delta E^{\prime}$.

true if the states are observed in any other inertial frame.

\section{Interconversion between different forms of energy}

Consider two states of a system. Observed in frame $S$, the first contains masses $m_{i}$ moving with velocities $\mathbf{u}_{i}$, but with the massless component bearing no energy. The second state consists of (i) masses $\widetilde{m}_{i}$ moving with velocities $\widetilde{\mathbf{u}}_{i}$, as well as (ii) a massless component which has energy $\Delta E$. In what follows, quantities observed in frame $S^{\prime}$ are primed.

Imposition of momentum conservation in frames $S, S^{\prime}$ yields, respectively,

$$
\sum_{i} m_{i} \mathbf{u}_{i}=\sum_{i} \widetilde{m}_{i} \widetilde{\mathbf{u}}_{i}
$$

and, for any $\mathbf{v}$,

$$
\sum_{i} m_{i}\left(\mathbf{u}_{i}-\mathbf{v}\right)=\sum_{i} \widetilde{m}_{i}\left(\widetilde{\mathbf{u}}_{i}-\mathbf{v}\right)
$$

which together imply that

$$
\sum_{i} m_{i}=\sum_{i} \widetilde{m}_{i}
$$

Thus, the conservation of momentum implies that no interconversion of rest energy into either kinetic energy or massless energy is possible.

Now imposing energy conservation in frames $S, S^{\prime}$, we obtain

$$
\frac{1}{2} \sum_{i} m_{i} u_{i}^{2}+\beta \sum_{i} m_{i}=\frac{1}{2} \sum_{i} \widetilde{m}_{i} \widetilde{u}_{i}^{2}+\beta \sum_{i} \widetilde{m}_{i}+\Delta E,
$$

and, for all $\mathbf{v}$,

$$
\begin{aligned}
\frac{1}{2} \sum_{i} m_{i}\left|\mathbf{u}_{i}-\mathbf{v}\right|^{2}+\beta \sum_{i} m_{i} & = \\
\frac{1}{2} \sum_{i} \widetilde{m}_{i}\left|\widetilde{\mathbf{u}}_{i}-\mathbf{v}\right|^{2} & +\beta \sum_{i} \widetilde{m}_{i}+\Delta E^{\prime},
\end{aligned}
$$

which, together with Eq. (10), imply that

$$
\Delta E^{\prime}=\Delta E .
$$


That is, the massless energy is frame-invariant ${ }^{16}$, and so does not transform in the same way as the total energy of the mass component, whose energy and momentum transform as:

$$
\begin{aligned}
& E^{\prime}=E-\mathbf{P} \cdot \mathbf{v}+\frac{1}{2} M v^{2} \\
& P^{\prime}=\mathbf{P}-M \mathbf{v}
\end{aligned}
$$

As we shall see later, this difference vanishes when one employs the Lorentz-rather than Galileantransformations to relate inertial frames.

Due to these results, the application of continuous conservation of energy and momentum conservation to an energetic system (consisting of mass- and masslesscomponents) proceeds as follows:

1. When imposing energy conservation to states involving a massless component, one must take into account the energy, $\Delta E$, of this component. That is, in frame $S$, Eq. (11) becomes

$$
\frac{1}{2} \sum_{i} m_{i} u_{i}^{2}=\frac{1}{2} \sum_{i} \widetilde{m}_{i} \widetilde{u}_{i}^{2}+\Delta E
$$

subject to total mass conservation, Eq. (10). And, in writing down the corresponding conservation statement in frame $S^{\prime}$, the energy of the massless component $\Delta E^{\prime}$ takes the same value as in $S$, namely $\Delta E$.

2. As previously discussed, the momentum of the massless component is presumed to be zero in all frames, so that one need only consider momenta associated with masses. That is, momentum is 'internally' continuously conserved.

\section{Frame-invariance equality of $(M, E, \mathbf{P})$}

Consider two systems, possibly containing differing numbers of particles in different states of motion. Let the systems be in states that, in frame $S$, are described by the tuples $(M, E, \mathbf{P})$ and $(\bar{M}, \bar{E}, \overline{\mathbf{P}})$. We show that, if these tuples are equal in frame $S$, then the corresponding tuples $\left(M^{\prime}, E^{\prime}, \mathbf{P}^{\prime}\right)$ and $\left(\bar{M}^{\prime}, \bar{E}^{\prime}, \overline{\mathbf{P}}^{\prime}\right)$ of the two systems as described in $S^{\prime}$ are also equal.

The preservation of equality of $M$ and $\bar{M}$ is immediate from the frame-invariance of total mass, Eq. (10). Con-

16 In Sec. IV B, we discuss a derivation of nonrelativistic kinetic energy due to Maimon in which the frame-invariance of massless energy is implicitly assumed. sider the quantities $E$ and $\mathbf{P}$ describing the first system,

$$
\begin{aligned}
& E=\frac{1}{2} \sum_{i} m_{i} u_{i}^{2}+\beta \sum m_{i}+\Delta E \\
& \mathbf{P}=\sum m_{i} \mathbf{u}_{i}
\end{aligned}
$$

where $\Delta E$ is the energy of the massless component.

Using Eq. (13), $E$ and $\mathbf{P}$ transform as

$$
\begin{aligned}
E^{\prime} & =\frac{1}{2} \sum_{i} m_{i}\left|\mathbf{u}_{i}-\mathbf{v}\right|^{2}+\beta \sum m_{i}+\Delta E^{\prime} \\
& =E-\mathbf{P} \cdot \mathbf{v}+\frac{1}{2} M v^{2}
\end{aligned}
$$

and

$$
\begin{aligned}
\mathbf{P}^{\prime} & =\sum m_{i}\left(\mathbf{u}_{i}-\mathbf{v}\right) \\
& =\mathbf{P}-M \mathbf{v}
\end{aligned}
$$

Thus, the tuple $\left(M^{\prime}, E^{\prime}, \mathbf{P}^{\prime}\right)$ is determined by $(M, E, \mathbf{P})$. It follows that, if $(M, E, \mathbf{P})=(\bar{M}, \bar{E}, \overline{\mathbf{P}})$, then the tuples $\left(M^{\prime}, E^{\prime}, \mathbf{P}^{\prime}\right)$ and $\left(\bar{M}^{\prime}, \bar{E}^{\prime}, \overline{\mathbf{P}}^{\prime}\right)$ of the systems observed in frame $S^{\prime}$ are also equal. Consequently, the tuple $(M, E, \mathbf{P})$ can be thought of as the macrostate of the energetic system (composed of a massive and massless component).

In particular, note that, as $\left(M^{\prime}, E^{\prime}, \mathbf{P}^{\prime}\right)$ explicitly depends upon $M$, one can find systems such that $(E, \mathbf{P})=$ $(\bar{E}, \overline{\mathbf{P}})$ but $\left(E^{\prime}, \mathbf{P}^{\prime}\right) \neq\left(\bar{E}^{\prime}, \overline{\mathbf{P}}^{\prime}\right)$. For example, consider two systems, each containing two particles of equal mass, moving at equal speeds in opposite directions along the $x$-axis. Let the first system contain particles of mass $m$, moving at speed $u$; and the second with particles of mass $m / 4$ moving at speed $2 u$. These systems have equal energy $\left(2 m u^{2}\right.$ ) and momentum (zero) in $S$, but unequal energy and momentum in $S^{\prime}$. In a relativistic framework, however, the equality of $(E, \mathbf{P})$ tuples of two systems is frame-invariant, irrespective of whether or not these systems have equal masses (see Sec. III B 2).

\section{Development of Newton's dynamical theory}

The development above is based on the consideration of collisions. However, a dynamical theory must allow for interactions between bodies even when separated, and further allow for ongoing changes in motion. To build such a theory, one requires an explicit model for motionchange which is sufficiently broad as to be applicable to widely-separated bodies in ongoing interaction.

The simplest generalization of the previous collisionbased considerations is to assume that continuous interaction between, say, two bodies (that are, in gen- 
eral, separated from one another) can be arbitrarily well approximated by a staccato model in which each body suffers a rapid succession of small abrupt changes of its motion ${ }^{17}$. Between these changes - by the principle of inertia - these two bodies move at constant velocity. Due to relativity, one can - without loss of generalityconsider the effect of each body's change of motion in its initial rest frame. In a body's initial rest frame, $\bar{S}$, an abrupt change of motion causes the body, initially at rest, to move off at velocity $\Delta \overline{\mathbf{u}}$. Thus, the effect of the influence on the body's change of motion is completely characterized by $\Delta \overline{\mathbf{u}}$.

Now, over a small time interval, $\Delta t$, suppose that a body undergoes $n$ abrupt velocity changes $\Delta \overline{\mathbf{u}}^{(1)}, \Delta \overline{\mathbf{u}}^{(2)}, \ldots, \Delta \overline{\mathbf{u}}^{(n)}$, with the $i$ th change referred to frame $\bar{S}^{(i)}$ in which the body is at rest immediately prior to this change. Due to Galilean kinematics, the net velocity change, $\Delta \overline{\mathbf{u}}$, in the frame, $\bar{S}$, in which the body is at rest immediately prior all of these changes, is the sum of these velocity changes,

$$
\Delta \overline{\mathbf{u}}=\Delta \overline{\mathbf{u}}^{(1)}+\Delta \overline{\mathbf{u}}^{(2)}+\cdots+\Delta \overline{\mathbf{u}}^{(n)} .
$$

By Galilean kinematics, velocity changes are frameindependent. Thus, as viewed in the lab frame, $S$, the cumulative effect of these change is to cause the body to undergo a change in velocity from $\mathbf{u}$ to $\mathbf{u}+\Delta \overline{\mathbf{u}}$.

Now, in a two-body system, it follows from the conservation of momentum that, if one body undergoes abrupt velocity changes due to the influence of the other, then the other must undergo corresponding abrupt velocity changes. Therefore, the only time-dependent quantities that can be attributed to body $i$ between velocity changes are its position, $\mathbf{r}_{i}$, and velocity, $\mathbf{u}_{i}$. Over the interval $[t, t+\Delta t]$, one also can attribute an average acceleration $\mathbf{a}_{i}=\Delta \mathbf{u}_{i} / \Delta t$.

We now postulate that each body's average acceleration in $\Delta t$ is determined by the bodies' masses and their time-dependent properties at the instant prior to the velocity changes that occur during this interval. Since the bodies move inertially between velocity jumps, these time-dependent properties consist in the bodies' positions and velocities only. Hence,

$$
\mathbf{a}_{1}=\mathbf{f}_{12}\left(m_{1}, m_{2} ; \mathbf{r}_{1}, \mathbf{r}_{2} ; \mathbf{u}_{1}, \mathbf{u}_{2}\right)
$$

17 The argument given here is inspired by the derivation given in $[14, \S 2.1]$. and

$$
\mathbf{a}_{2}=\mathbf{f}_{21}\left(m_{1}, m_{2} ; \mathbf{r}_{1}, \mathbf{r}_{2} ; \mathbf{u}_{1}, \mathbf{u}_{2}\right) .
$$

Here, the influence function $\mathbf{f}_{i j}$ encodes the influence on body $i$ due to body $j$. As previously shown (Eq. (10)), total mass is conserved. Here we additionally assume that the $m_{i}$ of separated particles remain constant during the interval.

Since the velocity change of each body takes the same value for two inertial frames in uniform relative motion, the $\mathbf{f}_{i j}$ must be do so also. Hence the latter can depend only on the bodies' frame-independent intrinsic properties, $m_{1}, m_{2}$, together with their relative position, $\mathbf{r}_{12} \equiv$ $\mathbf{r}_{2}-\mathbf{r}_{1}$, and relative velocity, $\mathbf{u}_{12} \equiv \mathbf{u}_{2}-\mathbf{u}_{1}$. Furthermore, due to the momentum conservation, namely

$$
m_{1} \Delta \mathbf{u}_{1}+m_{2} \Delta \mathbf{u}_{2}=\mathbf{0},
$$

the influence functions must satisfy the constraint

$$
m_{1} \mathbf{f}_{12}+m_{2} \mathbf{f}_{21}=\mathbf{0} .
$$

If one defines the force functions $\mathbf{F}_{12}=\mathbf{f}_{12} / m_{1}$ and $\mathbf{F}_{21}=\mathbf{f}_{21} / m_{2}$, then the above constraint can be re-expressed in terms of the force functions,

$$
\mathbf{F}_{12}+\mathbf{F}_{21}=\mathbf{0},
$$

while the motion-change of body 1 can be expressed as

$$
m_{1} \mathbf{a}_{1}=\mathbf{F}_{12}\left(m_{1}, m_{2} ; \mathbf{r}_{12}, \mathbf{u}_{12}\right) \text {. }
$$

We can extend the above model to a system of three of more bodies by assuming that, during each interval $\Delta t$, a body suffers many small changes in velocity due to each of the other bodies considered separately. Due to Galilean kinematics, these velocity changes add vectorially. Thus, denoting the velocity change of body $i$ in interval $\Delta t$ due to the presence of body $j \neq i$ as $\Delta \mathbf{u}_{i}^{(j)}$, the actual velocity change of body $i$ due to the presence of all other bodies is

$$
\begin{aligned}
\Delta \mathbf{u}_{i} & =\sum_{i \neq j} \Delta \mathbf{u}_{i}^{(j)} \\
& =\sum_{i \neq j} \mathbf{F}_{i j}\left(m_{i}, m_{j} ; \mathbf{r}_{i j}, \mathbf{u}_{i j}\right) \Delta t / m_{i} .
\end{aligned}
$$

If one writes

$$
\mathbf{F}_{i}=m_{i} \Delta \mathbf{u}_{i} / \Delta t,
$$

where $\Delta \mathbf{u}_{i} / \Delta t$ is the average acceleration, this relation 
can alternatively be expressed as

$$
\mathbf{F}_{i}=\sum_{i \neq j} \mathbf{F}_{i j}\left(m_{i}, m_{j} ; \mathbf{r}_{i j}, \mathbf{u}_{i j}\right) .
$$

That is, the net force on body $i$ is the vector sum-for all $i \neq j$ - of the force exerted by $j$ on $i$.

One can further assert that the ideal of smooth motion change can be treated as the limit in which a body is subject to infinitesimally-small velocity jumps that are packed infinitely-densely in time. In that limit, the instantaneous acceleration $\mathbf{a}_{i}=\lim _{\Delta t \rightarrow 0} \Delta \mathbf{u}_{i} / \Delta t$, so that

$$
\mathbf{F}_{i}=m_{i} \mathbf{a}_{i}
$$

where $\mathbf{F}_{i}$ is determined through Eq. (30).

In summary, Newton's framework can be seen to arise the following assumptions (beyond those made earlier):

- Abruptness of change. Change in the motion of a body, due to interaction with another, is wellapproximated as arising through a rapid succession of small, abrupt velocity changes.

- Influence is a function of the $m_{i}, \mathbf{r}_{i}, \mathbf{u}_{i}$. In a twobody system, the change in velocity of a body in a given time-interval due to the influence of the other is a function of the bodies' positions, and velocities, together with their masses.

- Composition of influences. In a many-body system, the change in motion of a body is the resultant of that due to each of the other bodies considered separately.

More systematically:

- Newton's first law - the principle of inertia - is assumed in our approach. Alternatively, it can be regarded as a consequence of relativity plus the assumption of the permanence of rest (namely that, in some frame of reference, an isolated body once at rest for a finite time remains at rest).

- Newton's second law-viz. $\mathbf{F}_{i}=m_{i} \mathbf{a}_{i}$, with $\mathbf{F}_{i}$ a frame-independent function - then follows from

- the derived fact that total mass is conserved;

- the assumption that motion-change in a system of interacting bodies occurs via a rapid succession of abrupt changes;

- the above-mentioned assumptions concerning the functional form of two-body influence, and the composition of influences in a many-body system;
- the additivity of velocity changes, and their frame-independence, both of which follow from Galilean kinematics.

- Newton's third law-viz. $\mathbf{F}_{12}=-\mathbf{F}_{21}$ in a twobody system - then follows from

- the assumption of continuous conservation of a vectorial quantity of motion for the mass component of the system;

- the derived form of this vectorial quantity;

- the assumption that the massless component of an energetic system bears no momentum.

- The vectorial composition of forces is a restatement of the assumption of composition of influences given above, the latter being expressed in terms of the composition of velocity changes (which are additive due to Galilean kinematics).

- The expression for the 'work done by a force' $\mathbf{F} \cdot d \mathbf{x}$, which is needed to embed the Newtonian framework in the broader energetic framework, then follows from

- the assumption of asymptotic conservation of a scalar quantity of motion for the mass component of the system;

- the derived form of this scalar quantity.

We conclude with some remarks:

1. Momentum conservation as a basis for a dissipative dynamical theory. As we have shown above, continuous conservation of momentum applied to the energetic framework only requires that one take into account the momenta of the masses. Thus, it is possible to formulate a dissipative dynamical theory - namely one that allows for inelastic processes - to be based around momentum conservation which does not explicitly describe - or keep track of - the massless component of the system.

2. Energy conservation as a basis for non-dissipative dynamical theory. However, such a dissipative dynamical theory cannot be based on energy conservation. Hence, a dynamical theory based around energy conservation which does not track the massless component must restrict itself to processes in which the massless energy is a function of the state of the massive component. Thus, such a theory necessarily restricts itself to non-dissipative processes.

3. Interpretation of Newton's second law. It has often been asserted that Newton's second law is simply a 
definition of force, apparently meaning that the dynamical measure of force (viz. the rate of change of momentum) specified by the law contains no new physical content ${ }^{18}$. However, from the vantage point of the derivation above, the second law arises from a staccato model of motion-change wherein the motion of a body changes abruptly due to the influence of other bodies in a system. It is this model, together with relativity, which implies that a function of the body's average rate of change of velocity (excluding any higher temporal derivatives of position) provides the measure of the influence exerted upon it. In the absence of such a simplifying model, the measure of influence could conceivably depend upon a finite number of temporal derivatives of $\mathbf{r}$.

\section{RELATIVISTIC MECHANICS}

\section{A. Conserved Quantities}

We first derive the forms of the energy and momentum of a particle by assuming asymptotic conservation of energy for an elastic collision.

\section{Kinetic \&5 Rest Energy}

In parallel to the nonrelativistic case (Sec. II A 1), we assume that a particle of mass $m$ with speed $u$ has a scalar quantity of motion $F(u)$, to which we henceforth refer as its energy. We assume that $m$ is a frame-invariant parameter, and that the total energy of a system of widely-separated particles is the sum of their separate energies.

Energy conservation for the collision of Fig. 1 as seen in frame $S^{\prime}$ implies that

$$
F(u \oplus v)+F(u \oplus-v)=2 F(w)
$$

where $w=\left[(u / \gamma(v))^{2}+v^{2}\right]^{1 / 2}$ and $\oplus$ denotes collinear relativistic velocity addition.

Defining function $\widetilde{F}$ via the relation $\widetilde{F}(\gamma(u))=F(u)$, and using the identities

$$
\begin{aligned}
\gamma(u \oplus v) & =\gamma(u) \gamma(v)\left[1+\frac{u v}{c^{2}}\right] \\
\gamma(w) & =\gamma(u) \gamma(v)
\end{aligned}
$$

\footnotetext{
18 See, for instance, [19, p. 901], [20, p. 60]. See also the discussion of Newtonian principles given in [21, Ch. 10] due to Poincaré (§8) and Painlevé (§9); and also [14, §6-7].
}

this conservation equation can be rewritten

$$
\widetilde{F}(x)+\widetilde{F}(y)=2 \widetilde{F}\left(\frac{x+y}{2}\right)
$$

where $x=\gamma(u \oplus v)$ and $y=\gamma(u \oplus-v)$. This is Jensen's functional equation, with general solution (see Appendix A 3)

$$
\widetilde{F}(x)=a x+b .
$$

Hence, for a particle of mass $m$, the conserved scalar quantity of motion is $F_{m}(u)=a(m) \gamma(u)+b(m)$, where $a(m)$ and $b(m)$ are undetermined functions of $m$.

To determine the forms of $a(m)$ and $b(m)$, write $F_{m}(u)=a(m)(\gamma(u)-1)+(a(m)+b(m))$, and consider the energy of a mass $m=m_{1}+m_{2}$. The energy can be computed in two different ways, which must agree: $F_{m}(u)=F_{m_{1}}(u)+F_{m_{2}}(u)$. Defining $c(m)=$ $a(m)+b(m)$, one thus obtains

$$
\begin{aligned}
& \left(a(m)-\left[a\left(m_{1}\right)+a\left(m_{2}\right)\right]\right)(\gamma(u)-1)+ \\
& \left(c(m)-\left[c\left(m_{1}\right)+c\left(m_{2}\right)\right]\right)=0 .
\end{aligned}
$$

Setting $u=0$ shows that $c(m)$ satisfies Cauchy's additivity equation. The case $u \neq 0$ then shows that $a(m)$ also satisfies the additivity equation. Hence, $a(m)=$ $a_{0} m$ and $c(m)=c_{0} m$, which imply $b(m)=b_{0} m$, where $a_{0}, b_{0}, c_{0}$ are all constants.

Correspondence with the non-relativistic expression for energy then requires that $a_{0}=c^{2}$, so that

$$
F(u)=\gamma(u) m c^{2}+b_{0} m
$$

A non-zero value of $b_{0}$ would imply that there were two distinct contributions to rest energy, namely $b_{0} m$ and $m c^{2}$. It does not seem possible to show that $b_{0}=0$ using considerations involving conservation and symme$\operatorname{try}^{19}$. However, as $b_{0}=0$ is empirically well-supported, we assume at this point that $b_{0}=0$ in order to avoid undue complexity in what follows.

\section{Momentum}

The most direct way to derive the form of relativistic momentum is via Schütz's argument. Consider masses $m_{i}$ moving in frame $S$ at velocities $\mathbf{u}_{i}$, which then collide elastically and separate to yield masses $m_{i}$ mov-

\footnotetext{
19 In Sec. IV E, we discuss an argument due to Einstein [22] which purports to show that $b_{0}=0$.
} 
ing at velocities $\widetilde{\mathbf{u}}_{i}$, with no massless energy. Energy conservation in frames $S, S^{\prime}$ yield

$$
\begin{aligned}
\sum_{i} \gamma\left(u_{i}\right) m_{i} c^{2} & =\sum_{i} \gamma\left(\tilde{u}_{i}\right) m_{i} c^{2} \\
\sum_{i} \gamma\left(u_{i}^{\prime}\right) m_{i} c^{2} & =\sum_{i} \gamma\left(\tilde{u}_{i}^{\prime}\right) m_{i} c^{2} .
\end{aligned}
$$

Using the relation $\gamma\left(u^{\prime}\right)=\gamma(u) \gamma(v)\left(1-u_{x} v / c^{2}\right)$, the latter can be rewritten

$$
\begin{aligned}
\gamma(v) \sum_{i} \gamma\left(u_{i}\right) & {\left[1-\frac{u_{i x} v}{c^{2}}\right] m_{i} c^{2} } \\
& =\gamma(v) \sum_{i} \gamma\left(\tilde{u}_{i}\right)\left[1-\frac{\tilde{u}_{i x} v}{c^{2}}\right] m_{i} c^{2}
\end{aligned}
$$

Using the former, one thus obtains

$$
\sum_{i} \gamma\left(u_{i}\right) m_{i} u_{i x}=\sum_{i} \gamma\left(\tilde{u}_{i}\right) m_{i} \tilde{u}_{i x}
$$

which is momentum conservation in the $x$-direction. Momentum conservation in the $y$ - and $z$-directions follows similarly by considering frame $S^{\prime}$ moving in those directions. Thus, the vectorial conserved quantity of motion of a particle of mass $m$ and velocity $\mathbf{u}$ is $\gamma(u) m \mathbf{u}$ up to a multiplicative constant. Requiring correspondence with the nonrelativistic momentum fixes this constant to unity.

\section{Photons}

The relationship, $E=p c$, between the energy, $E$, and momentum, p, of massless particles that travel at light speed can be derived as the limiting case of the expressions for energy and momentum of massive particles. The relationship between energy of such a particle (a 'photon') and the frequency of a light wave can be obtained by applying conservation and relativity to a process (a 'collision') in which two waves of equal frequency $f$ are incident along the $y$-axis, and then scatter without change of frequency, receding along a line inclined at angle $\theta$ to the line of incidence.

If one assumes that a luminous plane wave has associated particles, each of whose energy, $E$, is a function of the wave frequency, $f$, such that $E=H(f)$, and one then applies conservation of energy in frame $S^{\prime}$, taking the Doppler effect into account, one obtains the functional equation

$$
2 H(\gamma f)=H(\gamma(1-\beta \cos \theta) f)+H(\gamma(1+\beta \cos \theta) f),
$$

which holds for all $\beta, \theta$. This yields the solution $E=h f$, up to an additive constant, where $h$ is some constant.

\section{B. Continuous energy and momentum conservation in an energetic framework}

Let us now consider how to fit relativistic mechanics into the energetic framework. If we continue to assume that the massless component bears energy but not momentum, we run into an immediate problem. To see this, consider a system of masses $m_{i}$ moving at velocity $u_{i}$ (for simplicity, in one dimension) that interact and give rise to masses $\widetilde{m}_{i}$ moving at velocity $\widetilde{u}_{i}$. Conservation of momentum in frames $S$ and $S^{\prime}$ yields:

$$
\sum_{i} \gamma\left(u_{i}\right) m_{i} u_{i}=\sum_{i} \gamma\left(\widetilde{u}_{i}\right) \widetilde{m}_{i} \widetilde{u}_{i}
$$

and

$\sum_{i} \gamma\left(u_{i} \oplus-v\right) m_{i}\left(u_{i} \oplus-v\right)=\sum_{i} \gamma\left(\widetilde{u}_{i} \oplus-v\right) \widetilde{m}_{i}\left(\widetilde{u}_{i}, \oplus-v\right)$

which holds for any $\mathbf{v}$. The latter becomes

$$
\gamma(v) \sum_{i} \gamma\left(u_{i}\right) m_{i}\left(u_{i}-v\right)=\gamma(v) \sum_{i} \gamma\left(\widetilde{u}_{i}\right) \widetilde{m}_{i}\left(\widetilde{u}_{i}-v\right),
$$

which, via Eq. (42), implies that

$$
\sum_{i} \gamma\left(u_{i}\right) m_{i}=\sum_{i} \gamma\left(\widetilde{u}_{i}\right) \widetilde{m}_{i}
$$

That is, the total mass energy (rest energy plus kinetic energy) is conserved. This has two striking consequences:

1. In an elastic collision in which two equal bodies collide head on, momentum cannot be conserved at the stillpoint if the bodies' masses remain unchanged. That is, momentum is no longer continuously conserved.

2. If there is no additional contribution to a particle's rest energy apart from $m c^{2}$ (that is $b_{0}=0$ in Eq. (37)), the conversion of kinetic energy to massless energy is not possible.

The second of these consequences is at odds with the nonrelativistic case (where conversion from kinetic energy to massless energy is possible), and thus violates the minimal requirement of correspondence. In order to remove both of these difficulties, we modify the energetic framework so that the massless component can bear momentum as well as energy. This change restores the continuous conservation of momentum, and removes the second difficulty above. 


\section{Interconversion of energy and momentum between massive and massless forms}

Consider again a system of masses - now in three dimension, with velocities $\mathbf{u}_{i}$ in $S$-but allowing for a massless component that can bear momentum as well energy. Momentum conservation in frames $S, S^{\prime}$ yields

$$
\sum_{i} \gamma\left(u_{i}\right) m_{i} \mathbf{u}_{i}=\sum_{i} \gamma\left(\widetilde{u}_{i}\right) \widetilde{m}_{i} \widetilde{\mathbf{u}}_{i}+\Delta \mathbf{P}
$$

where $\Delta \mathbf{P}$ is the massless momentum, and

$$
\sum_{i} \gamma\left(u_{i}^{\prime}\right) m_{i} \mathbf{u}_{i}^{\prime}=\sum_{i} \gamma\left(\widetilde{u}_{i}^{\prime}\right) \widetilde{m}_{i} \widetilde{\mathbf{u}}_{i}^{\prime}+\Delta \mathbf{P}^{\prime}
$$

Energy conservation in frames $S$ and $S^{\prime}$ additionally yields

$$
\sum_{i} \gamma\left(u_{i}\right) m_{i} c^{2}=\sum_{i} \gamma\left(\widetilde{u}_{i}\right) \widetilde{m}_{i} c^{2}+\Delta E,
$$

and

$$
\sum_{i} \gamma\left(u_{i}^{\prime}\right) m_{i} c^{2}=\sum_{i} \gamma\left(\widetilde{u}_{i}^{\prime}\right) \widetilde{m}_{i} c^{2}+\Delta E^{\prime}
$$

Using the relation $\gamma\left(u^{\prime}\right)=\gamma(u) \gamma(v)\left(1-u_{x} v / c^{2}\right)$, the latter can be rewritten

$$
\begin{aligned}
& \gamma(v) \sum_{i} \gamma\left(u_{i}\right)\left[1-\frac{u_{i x} v}{c^{2}}\right] m_{i} c^{2}= \\
& \gamma(v) \sum_{i} \gamma\left(\widetilde{u}_{i}\right)\left[1-\frac{\widetilde{u}_{i x} v}{c^{2}}\right] \widetilde{m}_{i} c^{2}+\Delta E^{\prime}
\end{aligned}
$$

Using Eqs. (48) and (46), this reduces to

$$
\Delta E^{\prime}=\gamma(v)\left(\Delta E-v \Delta P_{x}\right) .
$$

Similarly, using the relations

$$
\begin{aligned}
& \gamma\left(u^{\prime}\right) u_{x}^{\prime}=\gamma(u) \gamma(v)\left(u_{x}-v\right) \\
& \gamma\left(u^{\prime}\right) u_{y}^{\prime}=\gamma(u) u_{y} \\
& \gamma\left(u^{\prime}\right) u_{z}^{\prime}=\gamma(u) u_{z}
\end{aligned}
$$

together with the Eqs. (46) and (48), Eq. (47) becomes

$$
\begin{aligned}
& \Delta P_{x}^{\prime}=\gamma(v)\left(\Delta P_{x}-\frac{v \Delta E}{c^{2}}\right) \\
& \Delta P_{y}^{\prime}=\Delta P_{y} \\
& \Delta P_{z}^{\prime}=\Delta P_{z} .
\end{aligned}
$$

Thus, the transition to the relativistic case - which involves change of the transformation group and allowing for massless momentum-brings about two major changes as compared with the nonrelativistic case. First, the energy, $\Delta E$, and momentum, $\Delta \mathbf{P}$, of the massless component of the system transform in precisely the same way as the energy and momentum of the masscomponent. Second, variability of the system's total mass is not disallowed, so that the conversion of rest energy to kinetic and massless energy is, in principle, possible.

\section{Frame-invariance equality of $(E, \mathbf{P})$}

Since the mass and massless components' energy and momentum transform in the same way, the total energy and momentum, $(E, \mathbf{P})$, of the energetic system transform according to Eqs. (51) and (53). Hence, two systems with equal values of $(E, \mathbf{P})$ in frame $S$ will also have equal values in frame $S^{\prime}$, even if they have unequal mass $^{20}$. Thus, $(E, \mathbf{P})$ can be regarded as the macrostate of a relativistic energetic system.

\section{Force and work in relativistic mechanics}

Unlike the case in nonrelativistic physics, the continuous conservation of momentum applied to the energetic framework in the relativistic context requires that one allow a form of momentum other than that associated with the masses. Consequently, it is not possible to formulate a dynamical theory of the masses without taking into account the larger energetic framework (in which the masses are embedded) and explicitly tracking the energy and momentum of the massless component of the energetic system.

Nevertheless, the notion of a force acting on a particle can be developed in a manner parallel to that presented in Sec. IIC. As in Sec. IIC, our analysis is based on the following model of motion change: (i) a body's response to an influence takes the form of a rapid succession of small abrupt changes of its motion; and (ii) the body moves at constant velocity in between these motion changes. Again, due to relativity, there is no loss of generality in considering the effect of a body's change of motion within its instantaneous rest frame, $\bar{S}$, so that the abrupt change is characterized by the body's change of velocity, $\Delta \overline{\mathbf{u}}$ in $\bar{S}$.

Now, suppose that, over a small time interval, $\Delta \tau$, referred to $\bar{S}$, the body undergoes $n$ velocity

20 As described in Sec. IV C, this fact is used as an axiom in the derivations of relativistic energy and momentum due to both Ehlers et al. [23] and to Lalan [24]. 
changes $\Delta \overline{\mathbf{u}}^{(2)}, \Delta \overline{\mathbf{u}}^{(2)}, \ldots, \Delta \overline{\mathbf{u}}^{(n)}$, with the $i$ th change referred to frame $\bar{S}^{(i)}$ in which the body is at rest immediately prior to this change. Due to the governing Lorentzian kinematics, the net velocity change, $\Delta \overline{\mathbf{u}}$, in the frame, $\bar{S}$, in which the body is at rest prior to all of these changes, is approximately the sum of these velocity changes,

$$
\Delta \overline{\mathbf{u}}=\Delta \overline{\mathbf{u}}^{(1)}+\Delta \overline{\mathbf{u}}^{(2)}+\cdots+\Delta \overline{\mathbf{u}}^{(n)}+\mathcal{O}\left(\delta^{3} / c^{2}\right),
$$

where $\delta$ is of the order of the $\Delta \bar{u}^{(i)}$, and $|\delta / c|<<1$. However, the error term vanishes in the limit where the $\Delta \bar{u}^{(i)} \rightarrow 0$. We will henceforth work in this limiting case, neglecting this error term. Specifically, we will suppose that, in frame $\bar{S}$, infinitesimal velocity changes $d \overline{\mathbf{u}}_{1}, d \overline{\mathbf{u}}_{2}, \ldots$ referred to instantaneous rest frames $\bar{S}^{(1)}, \bar{S}^{(2)}, \ldots$ combine additively to yield infinitesimal velocity change $d \overline{\mathbf{u}}$ (referred to frame $\bar{S}$ ) in the interval $d \tau$.

Accordingly, the influence on the body can be quantified instantaneously via the proper acceleration, $\overline{\mathbf{a}}=$ $d \overline{\mathbf{u}} / d \tau$, which can be transformed to give the acceleration in any other frame. The cause of the proper acceleration $\overline{\mathbf{a}}$ can then be posited as being due to an influence, $\overline{\mathbf{f}}$ on the body:

$$
\overline{\mathbf{f}}=\frac{d \overline{\mathbf{u}}}{d \tau},
$$

where $\overline{\mathbf{f}}$ is some heretofore unspecified function.

Above, the notion of influence has been quantified in the body's instantaneous rest frame, $\bar{S}$. This quantification is special in the sense that influences are additive in this frame due to the additivity of infinitesimal velocity changes, and furthermore suffices for dynamical predictions, provided $\overline{\mathbf{f}}$ is known. The quantification of influence in other frames, however, involves some degree of arbitrariness ${ }^{21}$. One choice is to simply posit that

$$
\mathbf{f}=\frac{d \mathbf{u}}{d t},
$$

where $\mathbf{f}$ is the influence on the body as observed in frame $S$. The transformational relation between $\overline{\mathbf{f}}$ and $\mathbf{f}$ is then determined by the kinematical transformation of acceleration between frames $\bar{S}$ and $S$. However, suppose that, in frame $S$, two bodies interact elastically, and that the resulting change of velocity occurs when the bodies are so close that the propagation of influ-

${ }^{21}$ Einstein alludes to the arbitrariness that is involved in extending 'force $=$ mass $\times$ acceleration' to the relativistic setting in $[25$, $\S 10]$. ence between them occurs virtually instantaneously. In that case, in an interval of time $\Delta t$ that includes the interaction, the change of momentum can be entirely attributed to the bodies. Thus, the bodies' average accelerations, $\mathbf{a}_{i}=\Delta \mathbf{u}_{i} / \Delta t$, are constrained by the conservation of momentum,

$$
m_{1} d\left(\gamma\left(u_{1}\right) \mathbf{u}_{1}\right)+m_{2} d\left(\gamma\left(u_{2}\right) \mathbf{u}_{2}\right)=\mathbf{0} .
$$

In order to harmonize the definition of influence (Eq. (56)) and the above constraint on accelerations due to conservation of momentum, one can instead choose to measure the influence on the body via $d \mathbf{p} / d t$, whose measure is given by the expression

$$
\mathbf{F}=\frac{d \mathbf{p}}{d t} .
$$

We can accordingly speak of a force-with dynamical measure $d \mathbf{p} / d t$ - acting on the body. In terms of force, the conservation of momentum - for the special case of elastic interaction between minimally-separated bodiesreduces to $\mathbf{F}_{1}+\mathbf{F}_{2}=\mathbf{0}$. Another reason, unrelated to the conservation of momentum, for working with force (rather than influence) is that it yields the correct $\mathbf{E}$ and $\mathbf{B}$ field transformations when Lorentz's force law is assumed to hold in all frames [26].

Nevertheless, the above choice between measuring influence via $\mathbf{f}$ or via $\mathbf{F}$ is nontrivial since, unlike the situation in nonrelativistic mechanics, one is not determined by the other ${ }^{22}$ given the body's rest mass, $m$.

In general, although one can measure the force on a particle through $\mathbf{F}=d \mathbf{p} / d t$, the lack of continuous conservation of total particle momentum means that there is no general analogue to Newton's third law. Note that this conclusion does not rest upon suppositions concerning the finite speed of motion of the massless component's momentum.

If a body is subject to influences due to many sources, then one can assert the composition of influence (in analogy to Newtonian mechanics). Then, in the instantaneous rest frame $\bar{S}$, due to the additivity of infinitesimal velocity changes due to each of these influences, the corresponding influences (due to each of the sources) add vectorially. Since force and influence coincide in $\bar{S}$, it follows that the corresponding (proper) forces in this frame also add vectorially.

Finally, it follows from the expressions for relativistic energy and momentum derived above that $\mathbf{F} \cdot d \mathbf{x}$ quan-

\footnotetext{
22 Specifically, $\mathbf{F}=\gamma^{3} m \mathbf{a}_{\|}+\gamma m \mathbf{a}_{\perp}$, while $\mathbf{f}=\mathbf{a}_{\|}+\mathbf{a}_{\perp}$. Thus, for a particle of given mass, the velocity $\mathbf{u}$ must be given in order to convert $\mathbf{F}$ to $\mathbf{f}$ or $\mathbf{f}$ to $\mathbf{F}$.
} 
tifies the increase in kinetic energy of a particle moving through $d \mathbf{x}$ as it is acted upon by force $\mathbf{F}$. A clearer understanding - which traces back more directly to the notions of conservation and relativity - would be desirable of why the same relation, namely $d T=\mathbf{F} \cdot d \mathbf{x}=$ $\mathbf{u} \cdot d \mathbf{p}$, holds irrespective of the transformation group by which relativity is implemented.

\section{PRINCIPLED DERIVATIONS OF QUANTITIES OF MOTION}

The notion that a body in motion has an associated quantity of motion dependent upon both its speed and its quantity of matter (henceforth referred to as 'mass', on the understanding that the Newtonian distinction between mass and weight is not implied) occurs as early as the fourteenth century in Buridan's penetrating analysis of the motion of projectiles and other bodies (such as ships and grindstones) ${ }^{23}$. Buridan first argued that, as a body moves through the air, the air acts to resist (rather than, as Aristotle asserted, to maintain) the motion of the body. He then remarks that, given two projectiles of identical external shape and material form but differing mass - say, a hollow brass sphere and a solid brass ball of identical size and outer appearance - moving at the same speed, the heavier projectile suffers less diminution in speed than the lighter ${ }^{24}$.

Buridan observes that this phenomenon can be explained if one assumes that each body has a quantity of motion, its impetus, an increasing function of both its mass and speed, and that it is this quantity that is degraded by air resistance. For, on the assumption that the resistance of a body depends on its external size, shape, and texture, but not its mass, the two projectiles would experience the same rate of diminution of their quantities of motion, but the heavier would suffer a lower rate of reduction in speed. He further asserts, presumably on the grounds of mathematical simplicity, that the impetus of a body is a linear function of its mass and speed, $m u$.

Descartes subsequently echoed Buridan's assertion that the quantity of motion is $m u$, which Newton and others subsequently vectorialized in order to handle inelastic collisions. As indicated in the Introduction, the first principled derivation of a quantity of motion-

23 See $[27,28]$ for illuminating discussions. Buridan's 'The impetus theory of projectile motion' (from 'Questions on the Eight Books of the Physics of Aristotle') is available in [29] (see particularly p. 275).

24 Buridan speculates that, if such resistance were entirely absent, a body would continue its motion indefinitely, and that such a condition might obtain with heavenly bodies. $m u^{2}$ - appears to have been due to Huygens, which was based on Galileo's law of free fall and on Torricelli's principle. The importance of removing the dependency on specific laws (such as Galileo's law of free fall) in favour of general principles was recognized, for example by Jean Bernoulli (leading to a submission to the Académie des Sciences in 1724), but not resolved ${ }^{25}$.

As far as we have been able to ascertain, the first systematic derivation of the expressions of both momentum and energy from broad symmetry principles (for example, those based on the concepts of conservation and relativity), rather than specific laws, did not appear until the start of the twentieth century-Mach [3] and Dugas [21], for example, in their historically-minded analyses of the development of mechanics, make no mention of such derivations. However, such derivations began to appear soon after the beginning of the twentieth century (see, for example, Ref. [30]), apparently spurred by Einstein's special theory of relativity. Similar derivations have continued to appear, with many variations, until the present day.

The common feature of these derivations is the use of the principle of relativity to view a mechanical situation (most commonly a collision) from two different, but physically equivalent, standpoints. However, these derivations differ in the additional main idea that they employ. For example, some assume the conservation of a scalar quantity of motion, while others introduce a principle that relates the total energy and/or momentum in different frames. Those derivations that consider a collision (rather than some other mechanical situation) differ in the particular collision that they consider-whether one-dimensional or two-dimensional; whether specially chosen (for example, possessing special symmetries) or not; whether elastic, inelastic, or completely inelastic. If inelastic, some additional considerations concerning nonmotive energy are involved.

Below, we briefly describe and analyze a few selected derivations of particular interest.

\section{A. Desloge (1976)}

Desloge $[31,32]$ considers elastic collisions similar to that which we have done, except the masses after collision recede from one another along any line. Specifically, identical particles approach one another from opposite directions at speed $u$ along a line of incidence represented

\footnotetext{
${ }^{25}$ For details of the broader context of Bernoulli's submission, see [7], Chapter 7. Bernoulli's alternative derivations are discussed in Chapter 8.
} 
by unit vector $\hat{\mathbf{n}}$, and emerge from their collision moving at their original speeds in opposite directions along a line of recession $\hat{\mathbf{n}}^{\prime}$; and all $u, \hat{\mathbf{n}}, \hat{\mathbf{n}}^{\prime}$ are possible. Rather than separately seeking a scalar conserved quantity which is a function of speed as we have done, Desloge seeks an additive function, $h$, of velocity. In a frame $S^{\prime}$ moving at velocity $\mathbf{v}$, he thus obtains

$$
h(\mathbf{v}+u \hat{\mathbf{n}})+h(\mathbf{v}-u \hat{\mathbf{n}})=h\left(\mathbf{v}+u \hat{\mathbf{n}}^{\prime}\right)+h\left(\mathbf{v}-u \hat{\mathbf{n}}^{\prime}\right),
$$

which is to hold for all $u, \hat{\mathbf{n}}, \hat{\mathbf{n}}^{\prime}, \mathbf{v}$.

This equation has a rather elegant geometric interpretation. Consider a sphere of radius $u$ with centre at $\mathbf{v}$. Then the sum of the $h$-values at a pair of antipodal points is the same as that at any other pair, and is also independent of the sphere's radius and centre. One could regard this equation as a variation of Jensen's functional equation. As such a view would lead one to expect, the general solution contains terms linear in the components of the vector argument. However, the general solution also contains a quadratic term, so that

$$
h(\mathbf{u})=a+\mathbf{b} \cdot \mathbf{u}+c u^{2},
$$

with arbitrary $a, \mathbf{b}, c$, whose values could depend upon particle properties.

Additional arguments show that $\mu \mathbf{u}$ and $a+\frac{1}{2} \mu u^{2}$ are separately conserved, where $a, \mu$ are particle parameters. The connection of $\mu$ to mass is made ([20], Chapter 8) by defining $\mu$ as the relative mass of a particle (so that mass is operationally measured via Weyl's procedure), but the relation of parameter $a$ to mass is not investigated. The treatment of relativistic quantities of motion is analogous.

Remarks. Compared with our approach, Desloge requires that one consider a more general collision (one with an arbitrary line of recession), as well as arbitrary relative direction of movement of frames $S, S^{\prime}$. Mathematically, the approach employs a functional equation whose solution is rather intricate (owing to the vector argument of the unknown function), and requires additional, lengthy arguments to pare down the number of particle parameters. The payoff of this greater complexity is (i) a derivation of both energy (up to an additive particle parameter, in the nonrelativistic case) and momentum via a single functional equation, and (ii) a demonstration that these are the only quantities of motion that are independently conserved in an elastic collision.

\section{B. Maimon's derivation of nonrelativistic kinetic energy (2011)}

Maimon's derivation ${ }^{26}$ of nonrelativistic kinetic energy is noteworthy as it considers an inelastic collision, specifically a completely inelastic head-on collision of equal masses moving at the equal speeds. An additive scalar conserved quantity is assumed to exist to which two types of contribution can occur - one due to mass (in which case it is assumed to be a function of speed), and the other a non-mass type referred to as 'heat'. The latter is implicitly taken to be frame-independent. When viewed in frames $S, S^{\prime}$, one obtains respectively

$$
\begin{gathered}
f(u)+f(u)=\Delta \\
f(u+v)+f(u-v)=2 f(v)+\Delta^{\prime} .
\end{gathered}
$$

Assuming $\Delta=\Delta^{\prime}$ (that is, quantity of 'heat' is frameindependent), one can eliminate $\Delta$ to obtain

$$
f(u+v)+f(u-v)=2 f(u)+2 f(v) .
$$

Although the author (correctly) guesses its solution, this equation is known as the quadratic functional equation ${ }^{27}$, and has general solution $f(u)=a u^{2}$. We remark that this solution lacks a rest energy term due to the inelastic nature of the collision that is considered.

Remarks. The derivation is brief and elegant, reducing to a well-known functional equation. However, the derivation cannot be immediately generalized to the relativistic case since the assumption that 'heat' is frameinvariant no longer holds true. This makes clear that the assumption is not as trivial as it may initially appear. As we show in Sec. II B 1, the fact that the quantity of 'heat' is frame-invariant in the nonrelativistic case can be derived by applying conservation and relativity to an energetic framework.

\section{Ehlers, Rindler, and Penrose (1965)}

The derivation of Ehlers et. al. [23] of relativistic and nonrelativistic energy is based not on a consideration of collisions, but on the following assumptions (that together constitute their Assumption II):

1. Direction-independence of energy of a two-particle system. The sum of the energies of a pair of equalmass particles approaching each other at equal and

\footnotetext{
26 See http://www.physics.stackexchange.com/questions/535/

27 See, for example, Ref. [33], Chapter 9
} 
opposite speeds along a line is independent of the direction of this line.

2. Frame-invariance of equality of total energy. If two such systems, differing only in their lines of approach, have equal energy, then that equality holds even when the systems are observed in another inertial frame.

The first of these assumptions follows from the general notion of the isotropy of space, so the real weight is borne by the second. The nontriviality of the second assumption can be seen by noting that the assumption fails if the two particles instead move in the same direction.

In any case, once these assumptions are granted, the authors consider two systems, each composed of two equal-mass particles approaching each other along a line at speed $u$, where the lines of approach are along the $x$ and $y$-axes. Equating the sum of the energies of these two systems as seen in a moving frame (speed $v$ ), they obtain (in the nonrelativistic case):

$$
f(u+v)+f(u-v)=2 f\left(\sqrt{u^{2}+v^{2}}\right),
$$

which is the same as our Eq. (1). The equation is solved by reduction to Jensen's equation by writing $E\left(w^{2}\right)=$ $f(w)$ and noting that $u^{2}+v^{2}=\frac{1}{2}\left(u_{1}^{\prime 2}+u_{2}^{\prime 2}\right)$, where $u_{1}=$ $u+v$ and $u_{2}=u-v$ :

$$
E\left(u_{1}^{\prime 2}\right)+E\left(u_{2}^{\prime 2}\right)=2 E\left(\frac{1}{2}\left(u_{1}^{\prime 2}+u_{2}^{\prime 2}\right)\right) .
$$

The relativistic case follows the same pattern.

Remarks. The derivation is based not on conservation, but on an assumption (frame-invariance of equality of total energy for a given mechanical situation) which does not appear to follow naturally from elementary considerations. As we point out in Secs. II B 2 and III B 2, this assumption can itself be obtained as a by-product of deriving mechanics within an energetic framework.

A similar derivation by Lalan [24] (discussed in [34, §24]) obtains expressions for relativistic energy and momentum of a particle from the assumption that, if two systems have the same energy and momentum in one frame, then they also have the same energy and momentum in any other inertial frame. Like Ehlers et. al., Lalan considers two systems, each consisting of pair of identical particles approaching each other at equal speeds, with the lines of approach along the $x$ - and $y$-axes. He thereby obtains separate functional equations for relativistic energy and momentum, which, rather than being solved explicitly, are shown to be consistent with the known expressions for these quantities.

\section{Sonego and Pin (2005)}

Sonego and Pin [35] consider two bodies colliding elastically in one dimension. No special symmetries are assumed. The kinetic energy of a body is taken to be a function $T(u)$ of its speed $u$, and the asymptotic conservation of total kinetic energy is assumed in frame $S$ :

$$
T\left(u_{1}\right)+T\left(u_{2}\right)=T\left(\tilde{u}_{1}\right)+T\left(\tilde{u}_{2}\right),
$$

where $u_{i}$ and $\tilde{u}_{i}$ are the pre- and post-collisional speeds of body $i$. Schütz's argument is then used to obtain an expression for the momentum, $p$, in terms of the unknown function $T$. The authors then assume that $d T=u d p$, which yields an equation that can be solved for $T$, for both the nonrelativistic and relativistic cases.

Remarks. The argument is innovative in its combination of Schütz's argument (to obtain momentum in terms of kinetic energy) with the positing of a relationship between momentum and energy as a way of fixing these quantities.

The main weakness of the argument is the lack of justification of the specific relation between $d T$ and $d p$ which is posited. The authors point out that this relation follows from $d T=F d x$, which they regard as axiomatic (as the definition of kinetic energy). But it is unclear why one should regard $d T=F d x$ as more fundamental than the relationship between, say, kinetic energy and speed, which one seeks to derive. Furthermore, as we have pointed out in Sec. III C, in view of the changes sustained by the expressions for energy and momentum in moving from nonrelativistic to relativistic mechanics, it is remarkable that $d T=F d x$ should hold in relativistic and nonrelativistic mechanics alike - we know of no simple argument for why this should be so.

\section{E. Einstein (1935)}

Einstein's derivation [22] considers an elastic collision of equal bodies which, in frame $S$, approach along a line at equal speed $u$, and recede along another line at speed $u$. He shows that, viewed in frame $S^{\prime}$, it follows from velocity addition formulae that:

$$
\begin{aligned}
\gamma\left(u_{1}^{\prime}\right)+\gamma\left(u_{2}^{\prime}\right) & =\gamma\left(\tilde{u}_{1}^{\prime}\right)+\gamma\left(\tilde{u}_{2}^{\prime}\right) \\
u_{1}^{\prime} \gamma\left(u_{1}^{\prime}\right)+u_{2}^{\prime} \gamma\left(u_{2}^{\prime}\right) & =\tilde{u}_{1}^{\prime} \gamma\left(\tilde{u}_{1}^{\prime}\right)+\tilde{u}_{2}^{\prime} \gamma\left(\tilde{u}_{2}^{\prime}\right) .
\end{aligned}
$$

On this basis, the quantities $(\gamma(u)-1) m c^{2}$ and $\gamma(u) m u$ are taken as the kinetic energy and momentum, respectively.

A second argument is then given which aims to show that the rest energy is (or can be taken to be) $m c^{2}$. A 
variation of the above collision is considered in which the bodies collide inelastically, with the kinetic energy lost in the collision presumed to result in an equal mass-increase of the two bodies.. Thus, in frame $S$, the bodies, each initially of mass $m$, approach, both moving at speed $u$; and then recede (now each of mass $\widetilde{m}$ ), both moving at speed $\tilde{u}$. Taking the rest energy of each mass to be $E_{0}$, the conservation of energy in frames $S$ and $S^{\prime}$ then yields:

$$
2 E_{0}+2 m c^{2}[\gamma(u)-1]=2 \widetilde{E}_{0}+2 \widetilde{m} c^{2}[\gamma(\tilde{u})-1]
$$

and

$$
\begin{aligned}
& 2 E_{0}+m c^{2}\left[\gamma\left(u_{1}^{\prime}\right)+\gamma\left(u_{2}^{\prime}\right)-2\right] \\
& \quad=2 \widetilde{E}_{0}+\widetilde{m} c^{2}\left[\gamma\left(\tilde{u}_{1}^{\prime}\right)+\gamma\left(\tilde{u}_{2}^{\prime}\right)-2\right] .
\end{aligned}
$$

Using the fact that $\gamma\left(u_{1}^{\prime}\right)+\gamma\left(u_{2}^{\prime}\right)=2 \gamma(u) \gamma(v)$ and $\gamma\left(\tilde{u}_{1}^{\prime}\right)+$ $\gamma\left(\tilde{u}_{2}^{\prime}\right)=2 \gamma(\tilde{u}) \gamma(v)$, these yield

$$
E_{0}-m c^{2}=\widetilde{E}_{0}-\widetilde{m} c^{2},
$$

from which it follows that the change in rest energy of each body, $\left(E_{0}-\widetilde{E}_{0}\right)$, is proportional to its change of mass, $(m-\widetilde{m})$. Einstein then argues that, since restenergy changes are only determined to within an additive constant, "one can stipulate that $E_{0}$ should vanish together with $m$ ", hence that $E_{0}=m c^{2}$.

Remarks. The first part of the argument is similar to that we have used, although the collision under consideration is more general, and explicit functional equations are not formulated. The second part of the argument presumes that conversion of kinetic energy to mass energy is possible. However, as we have seen in Sec. II B 1 and III B 1, whether or not this is the case depends on the form of the energy of a mass, and upon other assumptions concerning the wider energetic system; and is indeed not true in the nonrelativistic energetic framework. If the presumption is nevertheless granted (which risks inadvertently assuming what is to be proved), then the conclusion of the argument can be strengthened by using the result of Eq. (37), according to which $E_{0}=m c^{2}+b_{0} m$ for mass $m$. Insertion into Eq. (70) implies that either $b_{0}=0$ or $m=\widetilde{m}$. But, by hypothesis, the collision is inelastic, so that $m \neq \widetilde{m}$, which implies that only the former possibility $\left(b_{0}=0\right)$ survives. Thus, $E_{0}=m c^{2}$.

\section{STRUCTURE OF CLASSICAL MECHANICS}

\section{A. Overview}

In the previous sections, classical mechanics has been reconstructed in three distinct steps:
I. Derivation of the asymptotically conserved quantities of motion via conservation and relativity.

II. Construction of the energetic framework (motivated by continuous conservation of energy and momentum).

III. Construction of the force framework (motivated by treatment of continuous interaction between separated particles).

These steps - and the principles employed, and results obtained, therein - are summarized in Tables I and II.

\section{Classification and Explanatory Role of Physical Principles}

In order to clarify the structure of mechanics, and to facilitate the following discussion, Tables I and II employ the following classification of physical principles according to their explanatory role ${ }^{28}$ :

1. Uniformity Principles (U). A uniformity principle posits constancy of some property in a particularly simple case. As uniformity seems to demand little or no explanation (in comparison to non-uniformity), uniformity principles often have a grounding role in a theory. Examples of uniformity principles include the principle of inertia (describing the simple case of the motion of an isolated body), and the principle of indifference (uniform a priori probabilities) in Bayesian probability theory (which prescribes how to assignment a probability distribution when no specific information is available).

2. Equivalence Principles (EQ). An equivalence principle asserts that the same physical laws apply to physical phenomena observed from two or more different standpoints, or to a physical system placed in two or more different contexts. These principles enable one to explain what can happen by pointing to something else that can happen, Huygens' derivations of his laws of collision being an exemplar ${ }^{29}$. Examples of equivalence principles include Galileo's principle of relativity and Einstein's equivalence principle.

28 The classification given here is extracted from [36]. The full classification described therein contains additional types of principle which are not required in the present discussion.

29 For example, using Galilean relativity, one can explain what happens in an elastic head-on collision of equal bodies moving at unequal speeds $u_{1}, u_{2}$ in terms of what happens when those some bodies collide at equal speeds $\left(u_{1}+u_{2}\right) / 2$. See also footnote 3 . 
3. Eliminative Principles (EL). An eliminative principle asserts that not all conceivable physical states, pairs of states (at two different times), or processes are possible, and specifies a constraint that realizable states, pairs of states, or processes must satisfy. Examples of eliminative principles include the principles of conservation of energy and momentum, the principle of least action, and Pauli's exclusion principle ${ }^{30}$.

4. Compositional Principles (C). A compositional principle asserts that, at some level of description, the description of a larger entity is determined by the corresponding description of its components. Various entities can be referred to, such as systems, trajectories, and quantities (such as energy or action) associated therewith. Examples of compositional principles include the additivity of mass, the vector additivity of forces, and the quantum mechanical tensor product rule for composite systems.

5. Correspondence Principles (CR). A correspondence principle asserts that there exists some quantitative agreement between two theoretical models of the 'same' physical system, often in some limit or other special case. Examples of correspondence principles include the quantum mechanical average-value correspondence principle [37, 38], which posits that the expected value of certain quantum mechanical operator relations agree with the corresponding classical mechanical relations.

6. Special Principles (S). Miscellaneous special assumptions or principles that do not fall under any of the other categories. Examples of special principles include the specific collision assumed in Step I, and the specific model of motion-change posited in Step III.

In the case of mechanics, the principle of relativity (EQ1) posits how a given situation will appear to different observers without constraining what dynamical processes are possible, and is thus part of the kinematics. The principle of inertia (U1) and the specific collision (S1) assumed in Step I both assume the possibility of specific kinds of motion, the former concerning a single isolated body, the latter concerning two bodies interacting via a collision; and both are the basis for the dynamics. The conservation principles (EL1-3) and the explicit model of

30 The first principle acts as a constraint on which start- and endstates can be dynamically connected; the second as a constraint on allowable paths connecting given initial and final configurations; and the third as a constraint on allowable quantum numbers ('old' quantum theory) or on possible multiparticle states ('new' quantum theory). motion-change (s2) are both integral parts of the dynamics, but each has a different explanatory role: the first explains why certain conceivable (or describable) motions do not in fact occur (because they do not conserve certain quantities of motion), while the second goes further and explains why a system in given initial state unfolds in a specific manner given the influence (or force) functions.

The compositional principles (C1-5) enable the analysis of a system composed of many entities; or, conversely, the building-up of a larger system from subsystems.. For example, the composition of influences (C5) allows the instantaneous behaviour of a given particle in a system of $N$ particles to be explained in terms of the instantaneous behaviour of that particle when it is one component of $(N-1)$ two-particle systems.

Finally, in the derivation of relativistic mechanics, a special assumption (s3) concerning the rest energy of a body (which, in the present derivation, appears to be ultimately grounded by appeal to experiment) and a simple correspondence assumption (CR1), are additionally employed.

\section{B. Grounding Physical Theory in Symmetry Principles}

In his 'Unreasonable effectiveness of mathematics in the natural sciences' [39], Wigner posits a three-fold hierarchy in physics: events, laws of nature, and symmetry principles. In particular, just as the laws of physics express regularities in events, symmetry principles express regularities in laws of physics - in short, symmetry principles are meta-laws. From this perspective, the laws posited in a physical theory are more secure to the extent to which they can be traced to symmetry principles.

As sketched in the Introduction, the early development of mechanics was based on the key ideas of conservation and relativity, which are both symmetry principles (of type EL and EQ, respectively) ${ }^{31}$. However, in the process of their formalization and refinement, they acquired

\footnotetext{
31 For example, in the case of conservation of energy, the transformation under consideration is time evolution of the system; the 'object' transformed is the physical state of the system; and the equivalence relation between states is that they 'possess' the same total energy. The conservation principle thus posits that time evolution is a symmetry transformation of physical states with respect to this equivalence relation.
} 
I. Quantities of motion

Particles

Properties

mass $(m)$; position $(\mathbf{r})$, velocity $(\mathbf{u})$; scalar quantity of motion, $f_{m}(u)$
II. Energetic framework

Massive \& Massless Components

Particles: mass; position, velocity; energy $f_{m}(u)=\beta m+m u^{2} / 2$; momentum $m \mathbf{u}$

Massless component: energy, $\Delta E$

EL2\&3 Continuous conservation of total energy and momentum

C3 Additivity of energies of massive and massless components

C1 Additivity of mass

C2 Additivity of scalar quantities of motion

S1 Specific elastic collision

Results

Corpuscular energy: $\beta m+m u^{2} / 2$

Corpuscular momentum: $m \mathbf{u}$
EQ1 Relativity

III. Force framework

Massive \& Massless Components

Particles: mass; position, velocity; energy $f_{m}(u)=\beta m+m u^{2} / 2$; momentum $m \mathbf{u}$

Massless component: energy, $\Delta E$

U1 Principle of inertia

EQ1 Relativity

EL3 Continuous conservation of total momentum

C5 Composition of influences

S2 Abruptness model of motion-change \& concept of influence

For two bodies:

(i) $m \mathbf{a}_{\mathbf{i}}=\mathbf{F}_{i}\left(m_{1}, m_{2} ; \mathbf{r}_{1}, \mathbf{r}_{2} ; \dot{\mathbf{r}}_{1}, \dot{\mathbf{r}}_{2} ; \ldots\right)$, with force $\mathbf{F}_{i}$ frame-independent;

(ii) $\mathbf{F}_{1}+\mathbf{F}_{2}=\mathbf{0}$

Composition of forces $\left(\mathbf{F}_{i}=\sum_{i \neq j} \mathbf{F}_{i j}\right)$

Work-energy theorem $(d T=\mathbf{F} \cdot d \mathbf{x})$

Total system energy, $\Delta E+\sum \beta m_{i}+$ $m_{i} u_{i}^{2}$, and momentum, $\sum m_{i} \mathbf{u}_{i}$, are continuously conserved in all processes.

That total system energy be continuously conserved in an elastic collision.
That one be able to treat continuous interactions between separated bodies.

TABLE I: Structure of nonrelativistic mechanics. The derivation occurs in three distinct steps. In each step, the table summarizes (a) entities and their properties; (b) the principles and assumptions employed; (c) the main results, and (d) the key motivation. Each principle or assumption is preceded by a label (U, EQ, EL, C, CR, S) indicating the category of principle (uniformity, equivalence, eliminative, compositional, correspondence, special) to which it belongs - see Sec. V A 1followed by a number. The principles are numbered so as to emphasize the parallelism with the derivation of relativistic mechanics. As a consequence, one principle, namely C4 (additivity of momenta of the massive and massless components), is not used above. Note that the results of one step are incorporated into the following step (if one exists). The transition from Step I to Step II is driven by the desideratum that energy be continuously — not just asymptotically — conserved. The desire to treat continuous interactions between separated bodies drives the transition from Step II to Step III.

additions that were not obviously or clearly traced to symmetry principles.

Consider, for example, the formal principle of asymptotic conservation of energy, namely that $\sum m_{i} u_{i}^{2}$ is conserved under dynamical evolution of an isolated system of masses undergoing elastic contact interactions. The core of this principle - that a certain total 'quantity of motion' is conserved under a system's time evolutionis what one could regard as a pure symmetry principle. However, the quantitative part of this principle posits a specific quantity of motion, namely $\sum m_{i} u_{i}^{2}$. One can, in turn, split this quantitative assertion into two distinct statements:

1. Each body possesses a scalar quantity of motion $m_{i} u_{i}^{2}$.

2. The total quantity of motion is the sum of those of the individual bodies.

The first is a specific assertion. It is not a symmetry principle or obviously related to one. The second is a compositional principle, and also not explicitly traced back to a symmetry principle. This does not preclude these elements being separately derived from symmetry principles. But, taken in isolation, the principle is a hybrid 
I. Quantities of motion

Particles

Properties

mass $(m)$; position $(\mathbf{r})$, velocity $(\mathbf{u})$; scalar quantity of motion, $F_{m}(u)$

Principles \& Assumptions

U1 Principle of inertia

EQ1 Relativity

EL1 Asymptotic conservation of total scalar quantity of motion in elastic collision

C1 Additivity of mass

C2 Additivity of scalar quantities of motion

CR1 Correspondence of relativistic energy expression in the limit of small speeds

S1 Specific elastic collision

S3 Rest energy has no massindependent contribution

energy $\gamma(u) m c^{2}$;

momentum $\gamma(u) m \mathbf{u}$

Massless component:

energy, $\Delta E$; momentum, $\Delta \mathbf{P}$

EQ1 Relativity

EL2\&3 Continuous conservation of total energy and momentum

C3\&4 Additivity of energies and momenta of massive and massless components

\section{Force framework}

Massive \& Massless Components

Particles: mass; position, velocity; energy $\gamma(u) m c^{2}$; momentum $\gamma(u) m \mathbf{u}$

Massless component: energy, $\Delta E ;$ momentum, $\Delta \mathbf{P}$

U1 Principle of inertia

EQ1 Relativity

EL3 Continuous conservation of total momentum

C5 Composition of influences

S2 Abruptness model of motion-change \& concept of influence
Results

Corpuscular energy: $\gamma(u) m c^{2}$

Corpuscular momentum: $\gamma(u) m \mathbf{u}$

Massless energy and momentum transform between frames in the same manner as corpuscular energy and momentum

$d \mathbf{p} / d t=\mathbf{F}$, with force $\mathbf{F}$ framedependent;

Composition of forces $\left(\mathbf{F}_{i}=\sum_{i \neq j} \mathbf{F}_{i j}\right)$ holds in body's instantaneous rest frame

Work-energy theorem $(d T=\mathbf{F} \cdot d \mathbf{x})$

Remarks

Total corpuscular energy, $\sum \gamma\left(u_{i}\right) m_{i} c^{2}$, and momentum, $\sum \gamma\left(u_{i}\right) m_{i} \mathbf{u}_{i}$, are asymptotically conserved in elastic processes.

Motivations

That the sum total of a scalar quantity of motion be asymptotically conserved in an elastic collision.

\begin{abstract}
Total system energy, $\Delta E+\sum \gamma\left(u_{i}\right) m_{i} c^{2}$, and momentum, $\quad \Delta \mathbf{P}+\sum \gamma\left(u_{i}\right) m_{i} \mathbf{u}_{i}$, are continuously conserved in all processes.
\end{abstract}

That total system energy and momentum be continuously conserved in an elastic collision.
That one be able to treat continuous interactions between separated bodies.

TABLE II: Structure of relativistic mechanics. The derivation occurs in three distinct steps. In each step, the table summarizes (a) entities and their properties; (b) the principles and assumptions employed; (c) the main results, and (d) the key motivation. Each principle or assumption is preceded by a label (U, EQ, EL, C, CR, S) indicating the category of principle (uniformity, equivalence, eliminative, compositional, correspondence, special) to which it belongs - see Sec. V A 1-followed by a number. Note that the results of one step are incorporated into the following step (if one exists). The transition from Step I to Step II is driven by the desideratum that energy and momentum be continuously - not just asymptotically — conserved. The desire to treat continuous interactions between separated bodies drives the transition from Step II to Step III.

of two parts: a symmetry-based part, and a quantitative part $\left(\sum m_{i} u_{i}^{2}\right)$ that is not grounded on symmetry principles.

However, in Step I, it has been shown that the above conservation principle can - using relativity - be derived starting from a more austere basis, namely the asymptotic conservation of the sum total scalar quantity of motion, $\sum_{i} f_{m}\left(u_{i}\right)$, of a system of masses undergoing elastic collisions, provided that one assume that a specific collision is possible, and provided that one assume the additivity of mass and energy. In this manner, the above quantitative conservation principle is brought into closer contact with symmetry principles. The asymptotic conservation of momentum then follows immediately via a second application of relativity.

More generally, then, the process of grounding an existing physical theory, such as classical mechanics, on symmetry principles requires a careful re-examination of 
its mathematical principles, including those that might appear to be 'symmetry principles' but in fact contain elements that are not obviously grounded in symmetry.

As summarized in Tables I and II, it is possible to build up classical mechanics, guided by symmetry principles, in a fairly systematic fashion. The key symmetry principles employed are conservation (EL1-3) and relativity (EQ1), together with the principle of inertia (U1). However, in addition, the derivation employs the following special and compositional assumptions:

1. Three special assumptions, namely (i) a specific collision (S1) (as depicted in Fig. 1); (ii) a specific model of motion-change (S2) (Sec. II C); and (iii) in the relativistic case, the assumption that a particle's rest energy has no mass-independent contribution (S3) (Sec. III A 1).

2. Five compositional assumptions, namely (i) the additivity of mass (C1), (ii) the additivity of scalar quantities of motion (C2), (iii, iv) the additivity of energies of massive and massless components (C3\&4), and (v) the composition of influences (C5).

First, as mentioned in footnote 14, the specific collision can be largely justified on the basis of symmetry considerations. In contrast, the specific model of motion change is not based on a symmetry principle, but rather on the idea that continuous motion can be approximated by impulsive motion. Finally, in the relativistic case, it appears that assumption S3 is needed in order to rule out the possibility that a particle's rest energy has a contribution other than $m c^{2}$.

Second, compositional assumptions or principles lie in a separate category to symmetry principles, and yet seem to play as fundamental a role as symmetry principles in the building up of physical theories. Nevertheless, the mathematical form of certain compositional principles can be derived from symmetry considerations. For example, although the additivity of mass and the additivity of a scalar quantity of motion have been assumed ( $\mathrm{C} 1, \mathrm{C} 2)$, this additivity can, in fact, be derived from the symmetry of associativity. For example, if one assumes that the total mass of two bodies of mass $m_{1}, m_{2}$ is given by an unknown function $h\left(m_{1}, m_{2}\right)$, and one further requires that the mass of a system of three bodies can be determined by iteratively applying $h$ in a pairwise fashion, one notices that this composition can occur in either of two ways, either as $h\left(m_{1}, h\left(m_{2}, m_{3}\right)\right)$ or as $h\left(h\left(m_{1}, m_{2}\right), m_{3}\right)$. The requirement of associativity is that these two compositional pathways agree:

$$
h\left(m_{1}, h\left(m_{2}, m_{3}\right)\right)=h\left(h\left(m_{1}, m_{2}\right), m_{3}\right) .
$$

This functional equation, known as the associativity equation, implies that, without loss of generality, one can take $h$ to be the sum of its arguments ${ }^{32}$. Similarly, the vector addition of directed quantities of motion (as needed in C4), can be derived from elementary axioms following an argument originally due to d'Alembert ${ }^{33}$. In that derivation, basic symmetries, such as rotational covariance and commutativity \& associativity, play a leading role. Finally, as we have shown, it is possible to argue using relativity that, given the qualitative requirement that the total influence on a body is determined by the influence on that body due to each of the other bodies separately (C5), these influences (quantified as velocity changes) combine additively.

\section{Role of Conservation in Mechanics}

As sketched in the Introduction, the early historical development of mechanics (leading up to Newtonian mechanics) was marked by a striking conceptual tension between Descartes' notion of conservation - the idea that the total scalar 'quantity of motion' is conserved-and other considerations of similar intuitive force, most pointedly continuity (when applied to elastic collisions) and the presumption that 'hard' atoms undergo inelastic collisions. This tension was historically resolved through the marginalization of Descartes' notion of conservation in favor of the principle of conservation of momentum, culminating in Newton's theory of mechanics. Unlike Descartes' principle, the conservation of momentum was arrived at first and foremost as a mathematical transformation ('vectorialization') of Descartes' principle, and initially lacked intuitive justification of comparable force to Descartes' conservation principle. It was subsequently clothed in conceptual terms as being a consequence of the

\footnotetext{
32 More precisely, on the assumption that $h$ is differentiable at a point, one can show [40] that $h(a, b)=f^{-1}(f(a)+f(b))$, where $f$ is a continuous, monotonic function. Hence, if one regraduates the masses $m_{i}$ via $f$, so that $\mu_{i} \equiv f\left(m_{i}\right)$, then $\mu=\mu_{1}+\mu_{2}$ is the total regraduated mass of the system of two bodies. However, since $f$ is monotonic, one can just as well quantify the 'amount of matter' via the $\mu_{i}$ rather than the $m_{i}$. Hence, without loss of generality, one can say that mass is additive. The same line of argument applies to any scalar quantity, such as kinetic energy, associated with the bodies, provided that one has clear physical ground for believing that the total quantity for a system of bodies is a function of the quantities associated with each of the bodies.

33 See, for instance, Ref. [41], Chapter 1. The core assumptions here are: (i) the resultant of two parallel forces has magnitude equal to the sum of the magnitudes of these forces, and points in the same direction; (ii) the resultant of a number of forces is commutative and associative; (iii) the resultant of two forces is rotationally covariant; (iv) the resultant of two equal forces varies continuously with the angle between these forces.
} 
'principle of action and reaction', a notion very different from Descartes'.

As previously described, a century and a half later, Descartes' notion of conservation - with modifications due to Huygens and Leibniz - was effectively resurrected to serve as an overarching principle to coordinate diverse interconversion phenomena. By the end of the nineteenth century, the energetic framework - by then extended, due to electromagnetism, to include massless momentumhad became established as an indispensable part of the theoretical landscape. Finally, Einstein's theory of relativity showed that energy and momentum conservation were, in fact, two sides of a single conservation law. In particular, Laue's theorem showed that, in an electromagnetic system, the energy and momentum of matter and of fields transform in the same manner, namely as a four-vector.

The derivation given in the previous sections offers a vantage point from which one can gain insights into the subtle issues which were faced in relation to conservation during the historical development of mechanics.

\section{Nonrelativistic case}

We first consider the nonrelativistic case:

\section{Principled derivation of scalar conserved quantity of} motion. As shown in Step I, one can posit Descartes' notion of conservation for the asymptotic states of an elastic collision and then, by appealing to the principle of relativity, derive the conserved scalar quantity of motion, $m u^{2} / 2$. Hence, the tension between Descartes' original hypothesis (that $m u$ is the scalar conserved quantity) and relativity - a tension recognized by Huygens - can be directly resolved, and leads to $m u^{2} / 2$ without recourse to extraneous physical laws or principles (such as Galileo's laws of freefall-see footnote 4 ).

2. Relationship between scalar and vector conservation. Another application of relativity (via Schütz's argument) then leads from asymptotic energy conservation to asymptotic momentum conservation for elastic collisions. Thus, in this special case, these two principles, which were historically given such strikingly different intuitive justifications, are, in fact, intimately related, the former - when combined with relativity - implying the latter. Moreover, we see that, as long as the principle of relativity is presupposed, asymptotic scalar conservation must be accompanied by asymptotic vector conservation. The converse, however, does not holdgiven the principle of relativity, asymptotic vector con- servation can exist without asymptotic scalar conservation.

3. Continuous energy conservation. In Step II, the introduction of an energetic framework-with its notion of a massless form of energy - makes it possible to then posit that energy conservation holds continuously. Momentum conservation can also be posited to hold continuously, but without any evident need to introduce massless momentum. Thus, the energetic framework resolves the tension between scalar conservation and the requirement of continuity - as initially envisaged by Leibniz, but not embraced until the 1830s and 1840s.

4. Co-existence of scalar and vector conservation, and their consequences. These two conservation principles then yield nontrivial consequences, namely (i) total mass conservation (which implies no interconversion of rest energy to other forms of energy) and (ii) the frame-invariance of massless energy. Thus:

(a) Once generalized within an energetic framework, momentum conservation no longer follows from energy conservation. Instead, the two conservation laws independently co-exist, each yielding important consequences.

(b) One of those consequences is that total mass is conserved, a fact that therefore does not need to be independently assumed (as was the case historically).

(c) The other consequence is that massless energy is fundamentally different from kinetic energy, and hence cannot (as Leibniz envisaged) be assumed to be due to the motion of microscopic particles in a nonrelativistic framework ${ }^{34}$.

5. Possibility of momentum-based dissipative mechanical theory. Since momentum conservation holds continuously in the energetic framework without the need to posit a massless form of momentum, it is possible use continuous momentum as a basis for a mechanical theory which allows for inelastic collisions, but which only explicitly tracks massive bodies. Such a theory is constructed in Step III by introducing a staccato model of motion change.

34 We note that this implies that the (nonrelativistic) kinetic theory of gases is inconsistent-insofar as 'heat' is regarded as a form of massless energy, it is frame-invariant, and so cannot be represented by the kinetic energy of a set of particles, which is not frame-invariant. 
6. Possibility of non-dissipative energy-based mechanical theory. Since continuous energy conservation requires a massless form of energy, a dissipative mechanical theory which only tracks massive bodies cannot be build around continuous energy conservation. However, a non-dissipative (conservative) theory of such a type is possible.

\section{Relativistic case}

In the relativistic case:

1. Principled derivation of relativistic energy and momentum. Step I generalizes fairly straightforwardly from the nonrelativistic case, yielding the corresponding relativistic expressions for energy and momentum. Noteworthy here is the fact that:

(a) the expression for relativistic energy includes a rest energy component, $m c^{2}$; and

(b) one must assume that there is no contribution to a particle's rest energy other than $m c^{2}$.

2. Massless energy and momentum. In Step 2, the introduction of a massless form of momentum is essential in order to allow the conversion of kinetic energy to massless energy. Thus, the energetic framework must posit both massless energy and momentum. This stands against the historical development, in which it took the discovery of electromagnetic momentum to trigger the realization that momentum could be carried by something other than corpuscles.

3. Nature of massless energy-momentum. The generalized principles of conservation of energy and momentum then jointly imply that massless energymomentum transforms as a four-vector, and hence transforms in the same way as massive energymomentum. Thus, unlike the historical development, where the energy-momentum transformations laws were derived by consideration of the stressenergy-momentum tensor of an electromagnetic system [16] (see also [17, 18]), we see that the conservation laws directly imply that massless and massive energy-momentum have the same transformation laws; and they do so very generally since there is no need to specify any particular model of the massless component.

4. Interconversion of energy-momentum. As the transformation laws for energy and momentum are the same for the massless and massive components, the exchange of energy-momentum between these components is possible. In this connection, we note that, in contrast to the nonrelativistic case, a kinetic theory of gases is thereby rendered consistent.

\section{DISCUSSION}

\section{A. The relationship between symmetry transformations and conservation laws.}

Noether's theorems establish a connection between symmetry transformations and conservation laws. This is typically taken to be the ground for such assertions as 'invariance under temporal displacement underlies the conservation of energy'. However, as pointed out in [42], the connections between specific symmetry transformations and specific conservation laws (say, between temporal displacement and conservation of energy) presume the specific form of the action for a mechanical system ${ }^{35}$. As this form is conventionally obtained by requiring that the Euler-Lagrange equations of motion agree with those of Newtonian mechanics ${ }^{36}$, such assertions presume the latter.

The approach given here provides another way of seeing the connection between symmetry principles (which we take to include both the principle of relativity and conservation principles) and the quantities of mo-

35 We give here the some of the relevant quotes from [42]: "The conserved quantities of classical mechanics are Noether charges only because the classical equations of motion are what they are. But whether or not the classical equations of motion hold is something that needs to be established...". And: "Given what the equations of motion are, and that they hold where they do, it is indeed necessary that the conservation laws hold, but that's just a conditional necessity. The connection between the symmetries of the equations of motion and conservation laws is shown by Noether's theorem. That these are the correct equations of motion, however, is a completely different matter."

36 The assumptions underlying the least-action approach to nonrelativistic particle mechanics can be broken down as follows: (i) the (configuration-space) trajectory, $x(t)$, of a particle system between times $t_{1}, t_{2}$ has an associated action $S[x(t)]$; (ii) the actual trajectory between given configurations at times $t_{1}, t_{2}$ is one that extremizes $S[x(t)]$; (iii) the action is given by the time integral of a function, $L$, of $x(t)$ and a finite number of temporal derivatives thereof; (iv) the function $L$ has the form $L=T-V$, where $T, V$ are the kinetic and potential energies of the system. Of these assumptions, the first three can be posited independently from Newton's equations of motion. However, the common view is that the fourth-L $=T-V$-arises through a transformation of Newton's equations of motion via d'Alembert's principle (a more direct approach is given in [43]). Although it is possible to use fundamental symmetries (homogeneity of space and time, isotropy of space, and Galilean invariance) to show that $L$ is proportional to $T$ for a single isolated particle $[44, \S 4]$; and, further, to use compositional symmetries to show that $L=\sum_{i} T_{i}$ for a set of noninteracting particles, we are not aware of a derivation of $L=T-V$ that avoids presuming Newton's equations of motion. 
tion (and their relation with their corresponding massless forms). Specifically, one can see how the two symmetry principles interweave to produce the quantities of motion, and then shape the equations of motion. This is a rather different connection from that suggested by an application of Noether's theorem, but are perhaps more fundamental in the sense that the considerations given here precede the equations of motion (whereas an application of Noether's theorems to classical mechanics presupposes them).

\section{B. Pedagogical significance.}

The approach given here paves the way for a presentation of mechanics that unfolds systematically, guided primarily by symmetry principles. Such a presentation could have several benefits.

First, in typical presentations, nonrelativistic mechanics is presented via specific laws (Newton's laws plus the work-energy relationship), rather than via symmetry principles (such as conservation and relativity). The subsequent presentation of relativistic mechanics then demands a rather abrupt shift in thinking-style due to its reliance upon arguments that employ different frames of reference and conservation principles. But, in the approach given here, nonrelativistic and relativistic mechanics are developed in a parallel manner, guided by symmetry principles, with the continuity between them is clearly visible.

Second, almost all presentations of nonrelativistic mechanics introduces a number of specific laws axiomatically, whose interrelationship and basis is typically left unarticulated [45, 46]. For example, in [46], questions are mentioned such as:

(i) Is Newton's first law not simply a special case of the second?

(ii) Is Newton's second law a definition (of force), or a genuine law?

To these, one could add many others, such as:

(iii) Is the work-energy relationship (typically introduced axiomatically) a definition or a law? Is any other functional relation conceivable, or is the specific functional form derivable from some more fundamental basis?

(iv) why are there exactly two conserved quantities of motion, and why is one scalar and one vectorial?

(v) why is only one of the quantities of motion continuously conserved (if one only considers the quantities associated with the masses), while the other only asymptotically conserved (and then only for elastic collisions)?

As indicated in Sec. II and Sec. V C, all of these questions are naturally answered in the approach given here.

Analogously, the introduction of the Lorentz transformations to implement relativity brings about a cascade of changes in the dynamics - in addition to new expressions for corpuscular energy and momentum, one introduces massless momentum, and one finds that mass is no longer conserved. The question arises as to why (and to what extent) these changes in the dynamics are forced by the change in transformation group. In the usual presentations of relativistic dynamics, the connection between the dynamics and kinematics is obscured by a number of specific considerations, such as the use of the energyfrequency relationship of a photon. However, the connection is quite directly visible in the approach given here.

We also draw attention to the specific fact that the present approach explicitly shows that the same kind of considerations (involving relativity and conservation) that yield the expressions for particle energy and momentum also lead to the conclusion that the energy and momentum of the massless component of an energetic system transform in the same way as the energy and momentum of the massive component. In contrast, most presentations of relativistic dynamics restrict consideration to problems that involve only specific massive particles or photon-like particles, both of whose energies and momenta demonstrably transform as a four-vector. On the other hand, problems that involve massless energymomentum of unspecified form - such as where the annihilation of two massive particles gives rise to massless energy-momentum (of unspecified form) - are generally not considered, thereby side-stepping the question of whether the total energy-momentum of any system transforms as a four-vector.

Third, many of the above questions reflect often decades-long debates in the history of mechanics. For example, the relationship between the two distinct quantities of motion was a source of debate that persisted for more than a century [7, 8, 47]. Similarly, up until the end of the nineteenth century, concerns were repeatedly raised about the inclusion of the notion of force, itself not directly observable, in the foundations of mechanics, with attempts (such as that due to Hertz [48]) being made to reformulate classical mechanics without this notion. A symmetry-based presentation of mechanics puts these debates within touching distance. 


\section{Acknowledgments}

I am deeply grateful to Jennifer Coopersmith, Olivier Darrigol, Yiton Fu, Sebastiano Sonego, PierGianLuca Porta Mana, Costas Papachristou, and Sebastiano Sonego for discussion and feedback. I would particularly like to thank Olivier Darrigol for reading several versions of the paper, and for pointing out important historical omissions and logical errors, some of which led to substantial improvements.
[1] R. Descartes, Principles of Philosophy (Kluwer, 1982).

[2] R. S. Westfall, Force in Newton's Physics: The science of dynamics in the seventeenth century (Science History Publications, 1971).

[3] E. Mach, The Science of Mechanics: A Critical and Historical Account of Its Development (Open Court Pub. Co., 1919), 4th ed.

[4] J. B. Barbour, The Discovery of Dynamics (Oxford University Press, 2001).

[5] G. W. Leibniz, Acta eruditorum pp. 161-163 (1686), English translation, with supplements, in Philosophical papers and letters, 2nd edition, ed. L. E. Loemker, pp. 296302.

[6] W. L. Scott, The conflict between atomism and conservation theory 1644 to 1960 (Macdonald (London), Elsevier (NY), 1970).

[7] C. M. Iltis, Ph.D. thesis, University of Wisconsin, Madison (1967).

[8] C. M. Iltis, Studies in History and Philosophy of Science 1, 135 (1970).

[9] C. Wren, Phil. Trans. Roy. Soc. 3, 867 (1669).

[10] C. Huygens, Phil. Trans. Roy. Soc. 4, 925 (1669).

[11] C. Huygens, J. Scavans pp. 19-24 (1669).

[12] J. Wallis, Phil. Trans. Roy. Soc. 3, 864 (1669).

[13] T. S. Kuhn, in The essential tension, edited by T. S. Kuhn (University of Chicago Press, 1977).

[14] O. Darrigol, Archive for History of Exact Sciences (2019).

[15] J. R. Schütz, Nachr. v. d. Gesellschaft der Wissenschaften zu Göttingen, Math.-Phy. Kl. 2, 110 (1897).

[16] M. Laue, Ann. d. Phys 35, 524 (1911).

[17] D. Giulini, International Journal of Geometric Methods in Modern Physics 15, 1850182 (2018).

[18] H. C. Ohanian, Stud. Hist. Phil. Sci. 40, 167 (2009).

[19] H. Simon (1946).

[20] E. A. Desloge, Classical Mechanics., vol. 1 (John Wiley and Sons, Inc., 1982).

[21] R. Dugas, History of Mechanics (Dover Publications, 2011).

[22] A. Einstein, Bull. Amer. Math. Soc. 41, 223 (1935).

[23] J. Ehlers, W. Rindler, and R. Penrose, Am. J. Phys. 33, 995 (1965).

[24] M. V. Lalan, Comptes rendus de l'Académie des Sciences 198, 1211 (1934).

[25] A. Einstein, Ann. Phys. 17 (1905).

[26] M. Planck, Verhandlungen Deutsche Physikalische Gesellschaft 8, 136 (1906), English translation available at https://en.wikisource.org/wiki/Translation: The_Principle_of_Relativity_and_the_Fundamental_
Equations_of_Mechanics (accessed 22 Nov 2019).

[27] C. M. Graney, The Physics Teacher 51, 411 (2013).

[28] M. Clagett, The Science of Mechanics in the Middle Ages (University of Wisconsin Press, 1961).

[29] E. Grant, ed., A source book in Medieval science (Harvard University Press, 1974).

[30] R. C. Tolman, Phil. Mag. 23, 375 (1912).

[31] E. A. Desloge, Int. J. Theor. Phys. 15, 349 (1976).

[32] E. A. Desloge, Int. J. Theor. Phys. 15, 357 (1976).

[33] P. K. Sahoo and P. Kannappan, Introduction to Functional Equations (CRC Press, 2011).

[34] H. Arzeliès, Relativistic Point Dynamics (Pergamon Press, 1971).

[35] S. Sonego and M. Pin, Eur. J. Phys. 26, 33 (2005).

[36] P. Goyal, in preparation.

[37] P. Goyal, Phys. Rev. A 78, 052120 (2008).

[38] P. Goyal, arXiv:0910.2444 (2010).

[39] E. P. Wigner, Comm. in Pure and Applied Math 13, 1 (1960).

[40] J. Aczél, Lectures on Functional Equations and their Application (Academic Press, 1966).

[41] J. Aczél and J. Dhombres, Functional equations in several variables (Cambridge Univ. Press, 1989).

[42] J. Wolff, Phil. Sci. 8, 898 (2013).

[43] B. L. Beers, Am. J. Phys. 40, 1636 (1972).

[44] L. D. Landau and E. M. Lifshitz, Mechanics (Butterworth Heinemann, 2000), 3rd ed.

[45] C. Khiari, Lat. Am. J. Phys. Educ. 5 (2011).

[46] C. J. Papachristou, Nausivios Chora 4, 153 (2012).

[47] C. M. Iltis, Isis 62, 21 (1971).

[48] H. Hertz, The principles of mechanics presented in a new form (Macmillan \& Co., 1899), English translation. 


\section{Appendix A: Solution of Functional Equations}

In this appendix, the functional equations needed in the derivation of the energy and momentum of bodies are solved. If possible, we transform the functional equation of interest into a standard functional equation. For interest, we sometimes provide more than one possible method of solution. In each case, certain mathematical conditions must be satisfied by the unknown function in order for a solution to be obtained.

\section{Solution of $f(u+v)+f(u-v)=2 f\left(\sqrt{u^{2}+v^{2}}\right)$.}

We present two different solution methods for Eq. (1), one that transforms it into Jensen's functional equation, the other a direct solution by removing one degree of freedom.

a. Solution by transformation into Jensen's functional equation

Using the substitution $k\left(w^{2}\right)=f(w)$, Eq. (1) becomes

$$
k\left(\left[u^{2}+v^{2}\right]+2 u v\right)+k\left(\left[u^{2}+v^{2}\right]-2 u v\right)=2 k\left(u^{2}+v^{2}\right) .
$$

Setting $x=u^{2}+v^{2}, y=2 u v$, we obtain

$$
k(x+y)+k(x-y)=2 k(x)
$$

which is Jensen's equation, with $x, y$ independently variable within $x>0, y>0$. If $k$ is continuous, this equation, under the stated conditions, has general solution $k(z)=a z+b$. As $k$ is continuous whenever $f$ is continuous,

$$
f(v)=a v^{2}+b
$$

is the general solution of Eq. (1) under the condition that $f$ is continuous.

\section{b. Direct solution by removal of one degree of freedom}

Alternatively, one can directly solve Eq. (1) by removing one degree of freedom, albeit at the cost of the stronger regularity condition that $f$ is analytic. Setting $u=v$ in Eq. (1) yields

$$
f(2 u)+f(0)=2 f(\sqrt{2} u)
$$

If $f$ is differentiable, then, for $n \geq 1$,

$$
2^{n} f^{(n)}(2 u)=2^{1+n / 2} f(\sqrt{2} u)
$$

This yields $f^{(n)}(0)=0$ whenever $n \neq 2$. Hence, if $f$ is analytic,

$$
f(x)=a v^{2}+b .
$$

2. Solution of $g(v+u)-g(v-u)=2 g\left(\sqrt{u^{2}+v^{2}}\right) \cdot v / \sqrt{u^{2}+v^{2}}$.

Solution of Eq. (4) is most readily obtained by removing one degree of freedom by setting $v=u$. Thence,

$$
g(2 u)-g(0)=\sqrt{2} g(\sqrt{2} u)
$$


Setting $u=0$ fixes $g(0)=0$. If $g$ is differentiable, then, for $n \geq 1$,

$$
2^{n} g^{(n)}(2 u)=2^{(n+1) / 2} g^{(n)}(\sqrt{2} u) .
$$

For $n \geq 2$, this yields $g^{(n)}(0)=0$. Thus, if $g$ is analytic,

$$
g(u)=a u
$$

3. Solution of $\widetilde{F}(x)+\widetilde{F}(y)=2 \widetilde{F}((x+y) / 2)$.

Equation (34), with $x=\gamma(u \oplus-v)$ and $y=\gamma(u \oplus v)$, has the form of Jensen's equation, but it is not immediately apparent that $x, y$ are independent in some region. To see that this is so, it is helpful to express $u, v$ in terms of rapidities:

$$
\begin{aligned}
& u=c \tanh \phi_{1} \\
& v=c \tanh \phi_{2} .
\end{aligned}
$$

Then $u \oplus v=c \tanh \left(\phi_{1}+\phi_{2}\right)$, so that

$$
\begin{aligned}
\gamma(u \oplus v) & =\tilde{\gamma}\left(\phi_{1}+\phi_{2}\right) \\
\gamma(u \oplus-v) & =\tilde{\gamma}\left(\phi_{1}-\phi_{2}\right),
\end{aligned}
$$

where $\tilde{\gamma}(\phi) \equiv\left(1-\tanh ^{2} \phi\right)^{-1 / 2}$.

Now, $u>0$ and $|v|<c$, so that $\phi_{1}>0$ and $\phi_{2}$ is free. Consequently, $\left(\phi_{1}+\phi_{2}\right)$ and $\left(\phi_{1}-\phi_{2}\right)$ can be independently chosen. Further, since $\tilde{\gamma}$ is monotonic, $x=\tilde{\gamma}\left(\phi_{1}+\phi_{2}\right)$ and $y=\tilde{\gamma}\left(\phi_{1}-\phi_{2}\right)$ are independent in some region. Therefore, Eq. (34) has the solution $\widetilde{F}(x)=a+b x$. 